(C) 2003 American Chemical Society, Organometallics, Nakazawa om0208319 Supporting Info Page 1

\title{
C-C Bond Cleavage of Acetonitrile by a Carbonyl Iron Complex with a Silyl
}

\section{Ligand}

Hiroshi Nakazawa, ${ }^{\dagger \dagger}$ Takafumi Kawasaki; ${ }^{\ddagger}$ Katsuhiko Miyoshi, ${ }^{\ddagger}$ Cherumuttathu H. Suresh, ${ }^{\S}$ and Nobuaki Koga ${ }^{\S}$

Department of Chemistry, Graduate School of Science, Osaka City University, Sugimoto, Sumiyoshi-ku, Osaka 558-8585, Japan, Department of Chemistry, Graduate School of Science, Hiroshima University, Higashi-Hiroshima 739-8526, Japan, and Graduate School of Human Informatics and Venture Business Laboratory, Nagoya University, Chikusa-ku, Nagoya 464-8601, Japan

Supporting Information 


\section{X-ray Structure Reports for Complex 4}

\section{A. Crystal Data}

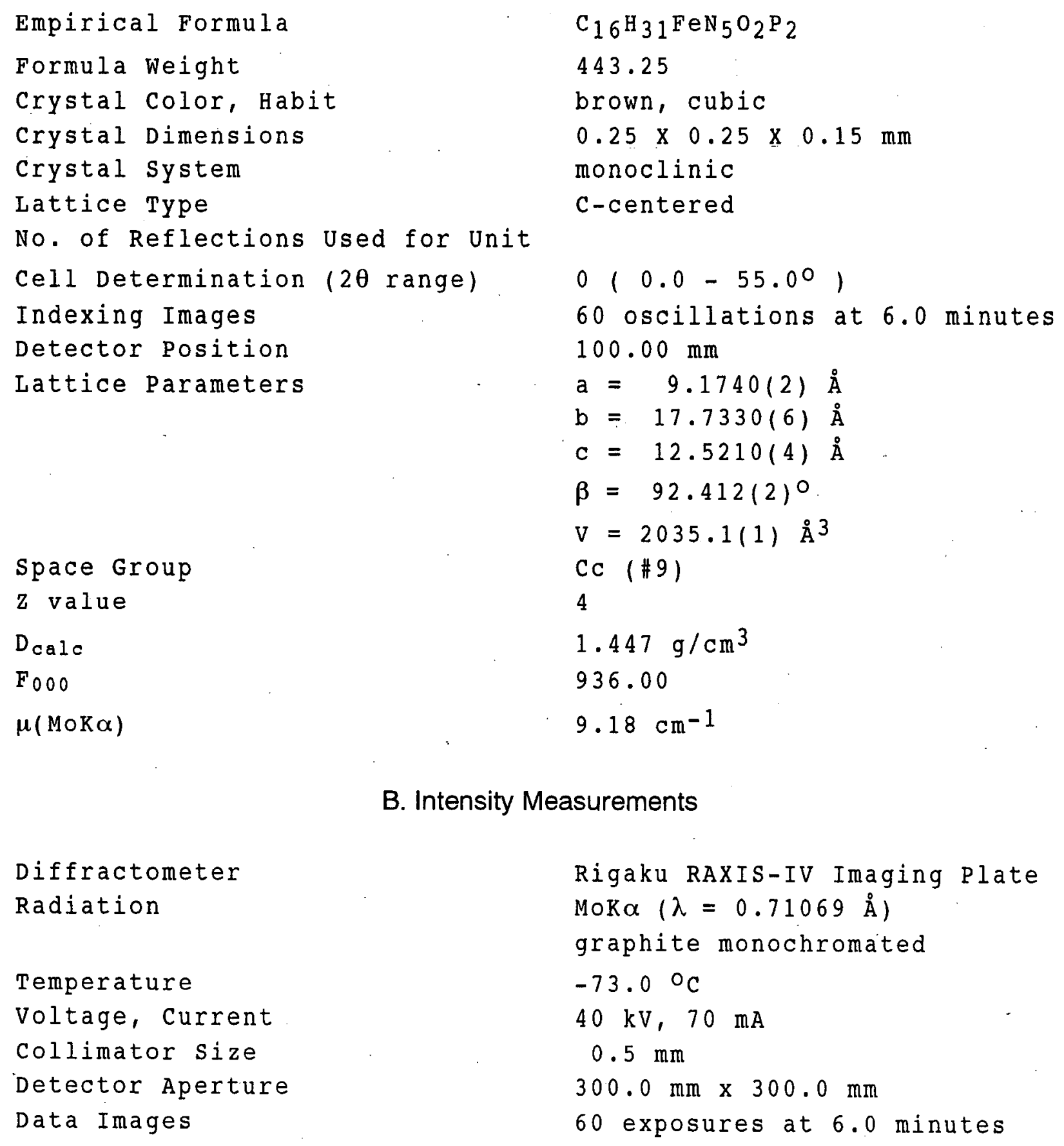


(C) 2003 American Chemical Society, Organometallics, Nakazawa om0208319 Supporting Info Page 3

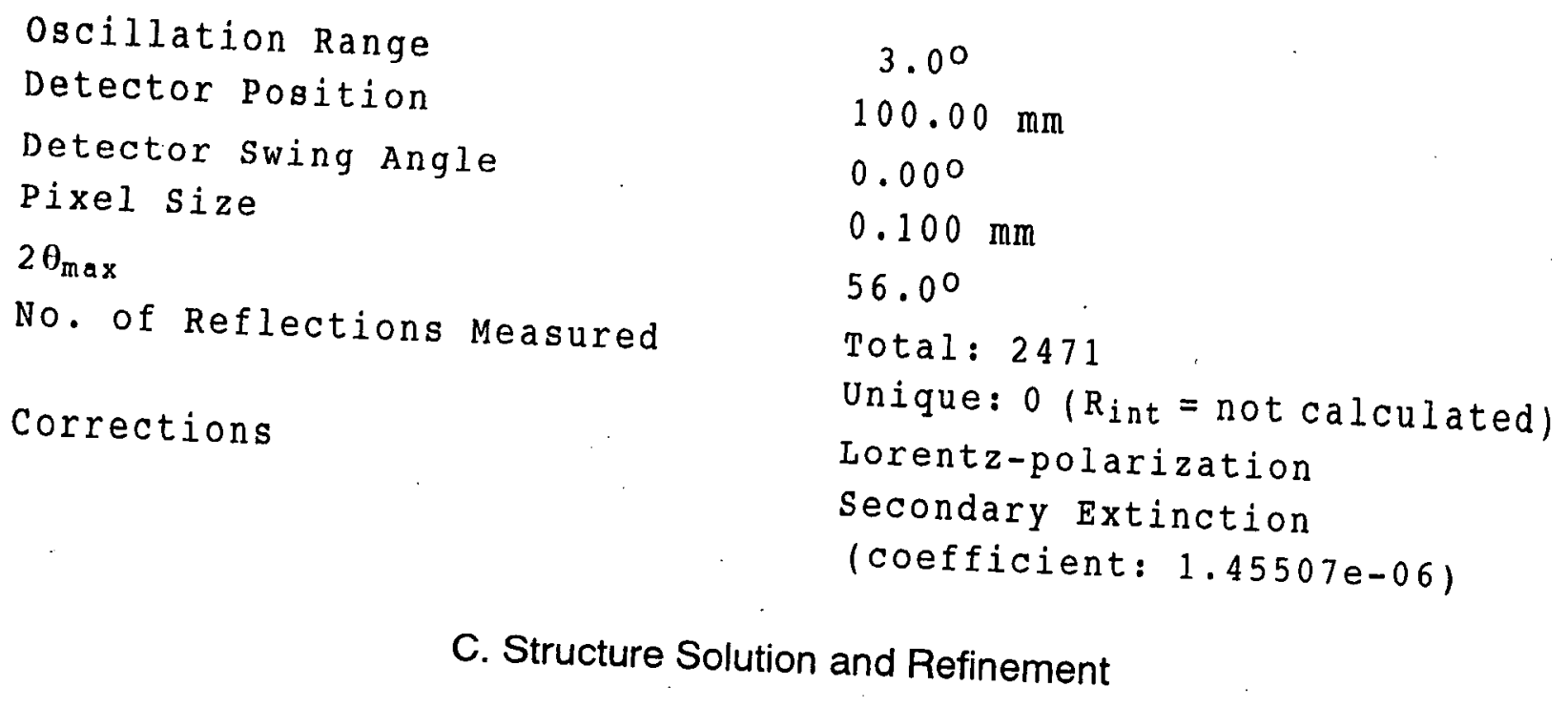

Structure solution
Refinement
Function Minimized

Least squares Weights

$p-f a c t o r$

Anomalous Dispersion

No. of observations

No. Variables

Reflection/Parameter Ratio

Residuals: $R$; $R W$

Residuals: $R 1$

No. of Reflections to calc R1

Goodness of Fit Indicator

Max Shift/Error in Final cycle

Maximum peak in Final Diff. Map

Minimum peak in Final Diff. Map
Direct Methods (SIR92)

Full-matrix least-squares

$\Sigma w(|F O|-|F C|)^{2}$

$1 / \sigma^{2}(\mathrm{FO})=4 \mathrm{FO}^{2} / \mathrm{\sigma}^{2}\left(\mathrm{FO}^{2}\right)$

0.0620

All non-hydrogen atoms

$$
\begin{aligned}
& 2 \theta<0.000,2354 \\
& 236 \\
& 9.97 \\
& 0.027 ; 0.043 \\
& 0.027 \\
& 2354 \\
& 1.31 \\
& 0.002 \\
& 0.32 \mathrm{e}^{-/ \AA^{3}} \\
& -0.54 \mathrm{e}^{-/ \AA^{3}}
\end{aligned}
$$


(C) 2003 American Chemical Society, Organometallics, Nakazawa om0208319 Supporting Info Page 4

Table 1. Atomic coordinates and $B_{i s o} / B_{\theta q}$

$\begin{array}{llcll}\text { atom } & \mathrm{x} & \mathrm{y} & \mathrm{z} & \mathrm{B} \\ \mathrm{Fe}(1) & 0.5961(8) & 0.16123(1) & 0.6470(6) & 1.013(6) \\ \mathrm{P}(1) & 0.7358(8) & 0.07315(3) & 0.5847(6) & 1.07(1) \\ \mathrm{P}(2) & 0.7153(8) & 0.25422(3) & 0.5822(6) & 1.14(1) \\ \text { O(1) } & 0.6941(8) & -0.00568(10) 0.6438(6) & 1.66(3) \\ \text { O(2) } & 0.8899(8) & 0.2392(1) & 0.5911(6) & 1.83(3) \\ \mathrm{N}(1) & 0.7668(9) & 0.1699(1) & 0.8626(6) & 2.20(5) \\ \text { N.(2) } & 0.9189(8) & 0.0719(1) & 0.5886(6) & 1.30(3) \\ \mathrm{N}(3) & 0.7282(8) & 0.0568(1) & 0.4523(6) & 1.58(3) \\ \text { N(4) } & 0.6868(9) & 0.3398(1) & 0.6362(6) & 1.99(5) \\ \text { N(5) } & 0.6824(8) & 0.2865(1) & 0.4577(6) & 1.67(4) \\ \text { C(1) } & 0.4012(9) & 0.1435(2) & 0.5566(6) & 2.57(5) \\ \text { C(2) } & 0.4114(9) & 0.0925(2) & 0.6447(7) & 2.55(5) \\ \text { C(3) } & 0.4186(9) & 0.1351(2) & 0.7394(6) & 2.54(5) \\ \text { C(4) } & 0.4154(9) & 0.2127(2) & 0.7116(7) & 2.60(5) \\ \text { C(5) } & 0.4044(9) & 0.2172(2) & 0.5991(7) & 2.67(5) \\ \text { C(6) } & 0.7106(9) & 0.1655(1) & 0.7776(6) & 1.38(4) \\ \text { C(7) } & 0.8703(8) & 0.0404(2) & 0.4084(6) & 2.03(5) \\ \text { C(8) } & 0.9799(9) & 0.0796(1) & 0.4832(6) & 1.92(4) \\ \text { C(9) } & 0.6019(9) & 0.0219(2) & 0.3988(6) & 2.47(5) \\ \text { C(10) } & 1.0113(9) & 0.0934(1) & 0.6808(6) & 1.73(4) \\ \text { C(11) } & 0.7670(9) & -0.0744(1) & 0.6183(6) & 2.13(5) \\ \text { C(12) } & 0.6312(9) & 0.3651(1) & 0.4536(6) & 2.10(4) \\ \text { C(13) } & 0.6886(9) & 0.4006(1) & 0.5572(7) & 2.35(5) \\ \text { C(14) } & 0.6210(9) & 0.2385(1) & 0.3723(6) & 1.94(5) \\ \text { C(15) } & 0.7340(9) & 0.3590(2) & 0.7440(7) & 3.29(7) \\ \text { C(16) } & 0.9924(9) & 0.2915(2) & 0.5530(7) & 3.40(7) \\ \text { H(1) } & 0.3909 & 0.1382 & 0.4721 & 3.5 \\ \text { H(2) } & 0.4250 & 0.0330 & 0.6454 & 3.8 \\ \text { H(3) } & 0.4499 & 0.0921 & 0.8220 & 4.0 \\ \text { H(4) } & 0.4391 & 0.2595 & 0.7748 & 2.8 \\ \text { H(5) } & 0.4320 & 0.2636 & 0.5474 & 6.7 \\ \text { H(6) } & 0.8917 & -0.0184 & 0.4066 & 3.1 \\ \text { H(7) } & 0.8768 & 0.0618 & 0.3433 & 1.1 \\ \text { H(8) } & 1.0807 & 0.0479 & 0.4870 & 4.0 \\ \text { H(9) } & 0.9882 & 0.1433 & 0.4675 & 2.5 \\ \text { H(10) } & 0.5121 & 0.0276 & 0.4399 & 5.4 \\ & & & & \end{array}$


(C) 2003 American Chemical Society, Organometallics, Nakazawa om0208319 Supporting Info Page 5

Table 1. Atomic coordinates and $B_{i s o / B} / B_{\text {eq }}$ (continued)

\begin{tabular}{|c|c|c|c|c|}
\hline a tom & $x$ & $y$ & $z$ & $B_{\theta q}$ \\
\hline $\mathrm{H}(11)$ & 0.6248 & -0.0320 & 0.3658 & 4.4 \\
\hline $\mathrm{H}(12)$ & 0.5865 & 0.0412 & 0.3257 & 6.1 \\
\hline $\mathrm{H}(13)$ & 0.9253 & 0.1062 & 0.7454 & 7.4 \\
\hline $\mathrm{H}(14)$ & 1.0933 & 0.0536 & 0.6932 & 2.0 \\
\hline $\mathrm{H}(15)$ & 1.0361 & 0.1296 & 0.6888 & 5.5 \\
\hline H $(16)$ & 0.7321 & -0.1184 & 0.6542 & 1.2 \\
\hline H (17) & 0.7532 & -0.0842 & 0.5391 & 2.9 \\
\hline $\mathrm{H}(18)$ & 0.8611 & -0.0699 & 0.6351 & 2.4 \\
\hline H (19) & 0.5191 & 0.3675 & 0.4405 & 2.0 \\
\hline $\mathrm{H}(20)$ & 0.6718 & 0.3878 & 0.3889 & 1.6 \\
\hline H $(21)$ & 0.6267 & 0.4405 & 0.5830 & 1.5 \\
\hline $\mathrm{H}(22)$ & 0.7907 & 0.4226 & 0.5557 & 2.9 \\
\hline$H(23)$ & 0.5091 & 0.2440 & 0.3764 & 3.9 \\
\hline $\mathrm{H}(24)$ & 0.6582 & 0.1771 & 0.3887 & 2.2 \\
\hline $\mathrm{H}(25)$ & 0.6605 & 0.2553 & 0.3059 & 0.8 \\
\hline $\mathrm{H}(26)$ & 0.7298 & 0.3078 & 0.8008 & 4.1 \\
\hline $\mathrm{H}(27)$ & 0.6778 & 0.4017 & 0.7849 & 5.2 \\
\hline $\mathrm{H}(28)$ & 0.8417 & 0.3749 & 0.7523 & 2.2 \\
\hline $\mathrm{H}(29)$ & 0.9470 & 0.2992 & 0.4740 & 7.3 \\
\hline $\mathrm{B}(30)$ & 1.0748 & 0.2760 & 0.5550 & 1.9 \\
\hline $\mathrm{H}(31)$ & 0.9703 & 0.3470 & 0.6050 & 5.8 \\
\hline
\end{tabular}


(C) 2003 American Chemical Society, Organometallics, Nakazawa om0208319 Supporting Info Page 6

Table 2. Anisotropic Displacement parameters

\begin{tabular}{|c|c|c|c|c|c|c|}
\hline m & $\mathrm{U}_{11}$ & $\mathrm{U}_{22}$ & $\mathrm{U}_{33}$ & $\mathrm{U}_{12}$ & $\mathrm{U}_{13}$ & $\mathrm{U}_{23}$ \\
\hline $\mathrm{Fe}(1)$ & $0.0108(2)$ & $0.0162(2)$ & $0.0116(2)$ & $0.0001(1)$ & $0.0013(1)$ & $09(1)$ \\
\hline$P(1)$ & $0.0137(3)$ & $0.0158(3)$ & $0.0110(2)$ & $-0.0001(2)$ & $0.0002(2)$. & $24(2)$ \\
\hline$P(2)$ & $.0130(3)$ & $0.0146(3)$ & $0.0155(3)$ & $-0.0001(2)-$ & $-0.0013(2)$ & $12(2)$ \\
\hline $0(1)$ & $.0274(9)$ & $0.0153(7)$ & $0.0204(8)$ & $-0.0026(6)$ & $0.0032(6)$ & $0.0009(6)$ \\
\hline$O(2)$ & (7) & $0.0227(8$ & $0.0344(9)$ & $0.0004(6)-$ & $-0.0023(6)$ & $0.0077(7)$ \\
\hline $\mathrm{N}(1)$ & $0.032(1)$ & $0.034(1)$ & $0.0165(9)$ & $-0.0007(9)-$ & $-0.0022(8)$ & $0.0006(8)$ \\
\hline (2) & $0.0147(8)$ & $0.0188(9)$ & $0.0161(8)$ & $0.0003(7)$ & $0.0008(6)$ & $-0.0007(6)$ \\
\hline $1(3)$ & $0.0211(9)$ & 0.02 & $0.0133(7)$ & $0.0035(7)$ & $-0.0018(7)-$ & $-0.0051(7)$ \\
\hline$N(4)$ & $0.033(1)$ & 0.01 & $0.029(1)$ & $0.0021(7)-$ & $-0.005(1)$ & $-0.0030(7)$ \\
\hline$N(5)$ & $0.024(1)$ & $0.0207(9)$ & $0.0182(8)$ & $0.0006(7)-$ & $-0.0017(7)$ & $0.0039(7)$ \\
\hline$C(1)$ & $0.008(1)$ & $0.062(1)$ & $0.028(1)$ & $-0.005(1)-$ & $-0.0034(8)$ & $-0.005(1)$ \\
\hline$c(2)$ & $0.014(1)$ & $0.032(1)$ & $0.052(2)$ & $-0.0057(9)$ & $0.004(1)$ & $-0.0035(.10)$ \\
\hline (3) & $0.010(1)$ & $0.054(1)$ & $0.034(1)$ & $-0.001(1)$ & $0.0105(9)$ & $0.013(1)$ \\
\hline$C(4)$ & $0.012(1)$ & $0.046(1)$ & $0.042(1)$ & $0.010(1)$ & $0.0071(9)-$ & $-0.012(1)$ \\
\hline$C(5)$ & $0.011(1)$ & $0.046(1)$ & $0.044(1)$ & $0.0075(10)$ & $0.0010(10)$ & $0.018(1)$ \\
\hline$c(6)$ & .019 & $0.019(1)$ & $0.0152(9)$ & $0.0025(7)$ & $0.0017(7)-$ & $-0.0002(7)$ \\
\hline$c(7)$ & $0.026(1)$ & 0.03 & 0.01 & $0.0092(9)$ & $0.0043(8)-$ & $-0.0052(8)$ \\
\hline$c(8)$ & $0.020(1)$ & $0.032(1)$ & 0.0 & $0.0056(8)$ & $0.0090(8)-$ & $-0.0015(8)$ \\
\hline$C(9)$ & $0.028(1)$ & $0.042(1)$ & $0.023(1)$ & $0.000(1)-$ & $-0.0084(9)-$ & $-0.015(1)$ \\
\hline$c(10)$ & $0.018(1)$ & $0.023(1)$ & $0.025(1)$ & $0.0026(8)-$ & $-0.0048(8)-$ & $-0.0027(8)$ \\
\hline$c(11)$ & $0.036(1)$ & $0.015(1)$ & $0.029(1)$ & $-0.0004(9)$ & $0.003(1)$ & $-0.0035(9)$ \\
\hline$c(12)$ & $0.025(1)$ & $0.022(1)$ & $0.032(1)$ & $0.0041(9)-$ & $-0.0026(9)$ & $0.0075(8)$ \\
\hline$C(13)$ & $0.037(1)$ & $0.018(1)$ & $0.034(1)$ & $0.0041(10)$ & $1-0.004(1)$ & $0.0037(8)$ \\
\hline$C(14)$ & $0.029(1)$ & $0.029(1)$ & $0.0152(10)$ & $0.0007(9)$ & $-0.0027(8)$ & $0.0012(8)$ \\
\hline$C(15)$ & $0.068(2)$ & $0.025(1)$ & $0.031(1)$ & $0.002(1)-$ & $-0.014(1)-$ & $-0.012(1)$ \\
\hline$c(16)$ & $0.013(1)$ & $0.035(1)$ & $0.081(2)$ & $-0.0077(10)$ & $0.002(1)$ & $0.024(1)$ \\
\hline
\end{tabular}

The general temperature factor expression:

$\exp \left(-2 P I^{2}\left(a * 2 U_{11} h^{2}+b * 2 U_{22} k^{2}+c * 2 U_{33} I^{2}+2 a * b * U_{12} h k+2 a * c * U_{13} h l+\right.\right.$ $\left.\left.2 b * C * U_{23} k 1\right)\right)$ 
(C) 2003 American Chemical Society, Organometallics, Nakazawa om0208319 Supporting Info Page 7

Table 3. Bond Lengths( $\AA)$

$\begin{array}{llllll}\text { atom } & \text { atom } & \text { distance } & \text { atom } & \text { atom } & \text { distance } \\ \mathrm{Fe}(1) & \mathrm{P}(1) & 2.1848(7) & \mathrm{Fe}(1) & \mathrm{P}(2) & 2.1558(7) \\ \mathrm{Fe}(1) & \mathrm{C}(1) & 2.100(3) & \mathrm{Fe}(1) & \mathrm{C}(2) & 2.087(3) \\ \mathrm{Fe}(1) & \mathrm{C}(3) & 2.089(3) & \mathrm{Fe}(1) & \mathrm{C}(4) & 2.086(3) \\ \mathrm{Fe}(1) & \mathrm{C}(5) & 2.086(3) & \mathrm{Fe}(1) & \mathrm{C}(6) & 1.907(3) \\ \mathrm{P}(1) & \mathrm{O}(1) & 1.635(2) & \mathrm{P}(1) & \mathrm{N}(2) & 1.678(2) \\ \mathrm{P}(1) & \mathrm{N}(3) & 1.682(2) & \mathrm{P}(2) & \mathrm{O}(2) & 1.623(2) \\ \mathrm{P}(2) & \mathrm{N}(4) & 1.686(3) & \mathrm{P}(2) & \mathrm{N}(5) & 1.675(2) \\ \mathrm{O}(1) & \mathrm{C}(11) & 1.432(3) & \mathrm{O}(2) & \mathrm{C}(16) & 1.417(4) \\ \mathrm{N}(1) & \mathrm{C}(6) & 1.166(4) & \mathrm{N}(2) & \mathrm{C}(8) & 1.462(3) \\ \mathrm{N}(2) & \mathrm{C}(10) & 1.454(3) & \mathrm{N}(3) & \mathrm{C}(7) & 1.465(4) \\ \mathrm{N}(3) & \mathrm{C}(9) & 1.452(4) & \mathrm{N}(4) & \mathrm{C}(13) & 1.464(4) \\ \mathrm{N}(4) & \mathrm{C}(15) & 1.441(5) & \mathrm{N}(5) & \mathrm{C}(12) & 1.471(4) \\ \mathrm{N}(5) & \mathrm{C}(14) & 1.461(4) & \mathrm{C}(1) & \mathrm{C}(2) & 1.426(5) \\ \mathrm{C}(1) & \mathrm{C}(5) & 1.412(5) & \mathrm{C}(2) & \mathrm{C}(3) & 1.405(5) \\ \mathrm{C}(3) & \mathrm{C}(4) & 1.419(5) & \mathrm{C}(4) & \mathrm{C}(5) & 1.411(5) \\ \mathrm{C}(7) & \mathrm{C}(8) & 1.514(4) & \mathrm{C}(12) & \mathrm{C}(13) & 1.517(4)\end{array}$


Table 4. Bond Lengths $(\AA)$

\begin{tabular}{|c|c|c|c|c|c|}
\hline atom & atom & distance & atom & atom & distance \\
\hline$c(1)$ & $\mathrm{H}(1)$ & 1.06 & $c(2)$ & $\mathrm{H}(2)$ & 1.06 \\
\hline$c(3)$ & $\mathrm{H}(3)$ & 1.31 & $C(4)$ & $\mathrm{H}(4)$ & $1: 16$ \\
\hline$c(5)$ & $\mathrm{H}(5)$ & 1.08 & $c(7)$ & $\mathrm{H}(6)$ & 1.06 \\
\hline$c(7)$ & $\mathrm{H}(7)$ & 0.90 & $c(8)$ & $\mathrm{H}(8)$ & 1.08 \\
\hline$c(8)$ & H (9) & 1.15 & $c(9)$ & $\mathrm{H}(10)$ & 0.99 \\
\hline$c(9)$ & $\mathrm{H}(11)$ & 1.07 & $c(9)$ & H $(12)$ & 0.98 \\
\hline$c(10)$ & $\mathrm{H}(13)$ & 1.18 & $c(10)$ & $\mathrm{H}(14)$ & 1.04 \\
\hline$c(10)$ & $\mathrm{H}(15)$ & 0.69 & $c(11)$ & Н $(16)$ & 0.96 \\
\hline$c(11)$ & $\mathrm{H}(17)$ & 1.01 & $c(11)$ & $\mathrm{H}(18)$ & 0.88 \\
\hline$c(12)$ & $\mathrm{H}(19)$ & 1.03 & $c(12)$ & $\mathrm{H}(20)$ & 0.99 \\
\hline$c(13)$ & $\mathrm{H}(21)$ & 0.97 & $c(13)$ & $\mathrm{H}(22)$ & 1.02 \\
\hline$C(14)$ & $\mathrm{H}(23)$ & 1.04 & $C(14)$ & $\mathrm{H}(24)$ & 1.16 \\
\hline$c(14)$ & Н $(25)$ & 0.97 & $c(15)$ & $\mathrm{H}(26)$ & 1.15 \\
\hline$c(15)$ & $\mathrm{H}(27)$ & 1.06 & $C(15)$ & $\mathrm{H}(28)$ & 1.03 \\
\hline$c(16)$ & H $(29)$. & 1.07 & $c(16)$ & $\mathrm{H}(30)$ & 0.80 \\
\hline$c(16)$ & $\mathrm{H}(31)$ & 1.20 & & & \\
\hline
\end{tabular}


Table 5. Bond Angles $\left(^{\circ}\right)$

\begin{tabular}{|c|c|c|c|c|c|c|c|}
\hline atom & atom & atom & angle & atom & atom & atom & angle \\
\hline$P(1)$ & $\mathrm{Fe}(1)$ & $P(2)$ & $95.64(3)$ & $P(1)$ & $\mathrm{Fe}(1)$ & $c(1)$ & $101.5(1)$ \\
\hline$P(1)$ & $\mathrm{Fe}(1)$ & $C(2)$ & $93.76(9)$ & $P(1)$ & $\mathrm{Fe}(1)$ & $c(3)$ & $121.4(1)$ \\
\hline$P(1)$ & $\mathrm{Fe}(1)$ & $c(4)$ & $159.9(1)$ & $P(1)$ & $\mathrm{Fe}(1)$ & $C(5)$ & $137.5(1)$ \\
\hline$P(1)$ & $\mathrm{Fe}(1)$ & $c(6)$ & $91.41(8)$ & $P(2)$ & $\mathrm{Fe}(1)$ & $c(1)$ & $110.2(1)$ \\
\hline$P(2)$ & $\mathrm{Fe}(1)$ & $c(2)$ & $150.0(1)$ & $P(2)$ & $\mathrm{Fe}(1)$ & $c(3)$ & $142.9(1)$ \\
\hline$P(2)$ & $\mathrm{Fe}(1)$ & $C(4)$ & $103.6(1)$ & $P(2)$ & $\mathrm{Fe}(1)$ & $C(5)$ & $87.84(9)$ \\
\hline$P(2)$ & $\mathrm{Fe}(1)$ & $c(6)$ & $91.28(8)$ & $c(1)$ & $\mathrm{Fe}(1)$ & $C(2)$ & $39.8(1)$ \\
\hline$c(1)$ & $\mathrm{Fe}(1)$ & $c(3)$ & $66.4(1)$ & $C(1)$ & $\mathrm{Fe}(1)$ & $c(4)$ & $66.5(1)$ \\
\hline$c(1)$ & $\mathrm{Fe}(1)$ & $c(5)$ & $39.4(2)$ & $C(1)$ & $\mathrm{Fe}(1)$ & $c(6)$ & $153.4(1)$ \\
\hline$c(2)$ & $\mathrm{Fe}(1)$ & $C(3)$ & $39.3(1)$ & $C(2)$ & $\mathrm{Fe}(1)$ & $C(4)$ & $66.6(1)$ \\
\hline$C(2)$ & $\mathrm{Fe}(1)$ & $c(5)$ & $66.4(1)$ & $c(2)$ & $\mathrm{Fe}(1)$ & $C(6)$ & $116.9(1)$ \\
\hline$c(3)$ & $\operatorname{Fe}(1)$ & $C(4)$ & $39.8(1)$ & $c(3)$ & $\mathrm{Fe}(1)$ & $c(5)$ & $66.3(1)$ \\
\hline$c(3)$ & $\mathrm{Fe}(1)$ & $c(6)$ & $87.0(1)$ & $C(4)$ & $\mathrm{Fe}(1)$ & $C(5)$ & $39.5(1)$ \\
\hline$c(4)$ & $\mathrm{Fe}(1)$ & $C(6)$ & $93.9(1)$ & $C(5)$ & $\mathrm{Fe}(1)$ & $c(6)$ & $130.9(1)$ \\
\hline $\mathrm{Fe}(1)$ & $P(1)$ & $0(1)$ & $107.34(8)$ & $\mathrm{Fe}(1)$ & $P(1)$. & $N(2)$ & $126.91(8)$ \\
\hline $\mathrm{Fe}(1)$ & $P(1)$ & $\mathrm{N}(3)$ & $118.34(9)$ & $0(1)$ & $\mathrm{P}(1)$ & $N(2)$ & $103.2(1)$ \\
\hline $0(1)$ & $\mathrm{P}(1)$ & $N(3)$ & $107.3(1)$ & $\mathrm{N}(2)$ & $\mathrm{P}(1)$ & $\mathrm{N}(3)$ & $91.5(1)$ \\
\hline $\mathrm{Fe}(1)$ & $P(2)$ & $0(2)$ & $111.31(8)$ & $\mathrm{Fe}(1)$ & $\mathrm{P}(2)$ & $\mathrm{N}(4)$ & $116.6(1)$ \\
\hline $\mathrm{Fe}(1)$ & $P(2)$ & $N(5)$ & $122.49(9)$ & $0(2)$ & $P(2)$ & $N(4)$ & $106.8(1)$ \\
\hline $0(2)$ & $P(2)$ & $N(5)$ & $105.0(1)$ & $N(4)$ & $P(2)$ & $N(5)$ & $92.4(1)$ \\
\hline$P(1)$ & $0(1)$ & $C(11)$ & $120.4(2)$ & $P(2)$ & $0(2)$ & $c(16)$ & $122.5(2)$ \\
\hline $\mathrm{P}(1)$ & $N(2)$ & $c(8)$ & $113.2(2)$ & $P(1)$ & $\mathrm{N}(2)$ & $C(10)$ & $124.7(2)$ \\
\hline$c(8)$ & $\mathrm{N}(2)$ & $c(10)$ & $117.3(2)$ & $P(1)$ & $N(3)$ & $c(7)$ & $113.8(2)$ \\
\hline$P(1)$ & $\mathrm{N}(3)$ & $C(9)$ & $121.8(2)$ & $c(7)$ & $N(3)$ & $c(9)$ & $116.6(2)$ \\
\hline$P(2)$ & $N(4)$ & $c(13)$ & $112.6(2)$ & $P(2)$ & $\mathrm{N}(4)$ & $c(15)$ & $122.9(2)$ \\
\hline$c(13)$ & $N(4)$ & $c(15)$ & $116.6(3)$ & $P(2)$ & $N(5)$ & $c(12)$ & $113.7(2)$ \\
\hline$P(2)$ & $N(5)$ & $C(14)$ & $122.1(2)$ & $C(12)$ & $N(5)$ & $C(14)$ & $114.4(2)$ \\
\hline $\mathrm{Fe}(1)$ & $C(1)$ & $C(2)$ & $69.6(2)$. & $\mathrm{Fe}(1)$ & $c(1)$ & $C(5)$ & $69.7(2)$ \\
\hline$C(2)$ & $C(1)$ & $C(5)$ & $107.2(3)$ & $\mathrm{Fe}(1)$ & $c(2)$ & $C(1)$ & $70.6(2)$ \\
\hline $\mathrm{Fe}(1)$ & $c(2)$ & $c(3)$ & $70.4(2)$ & $c(1)$ & $c(2)$ & $c(3)$ & $108.1(3)$ \\
\hline $\mathrm{Fe}(1)$ & $C(3)$ & $C(2)$ & $70.2(2)$ & $\mathrm{Fe}(1)$ & $C(3)$ & $C(4)$ & $70.0(2)$ \\
\hline$C(2)$ & $c(3)$ & $C(4)$ & $108.4(3)$ & $\mathrm{Fe}(1)$ & $C(4)$ & $c(3)$ & $70.2(2)$ \\
\hline $\mathrm{Fe}(1)$ & $C(4)$ & $C(5)$ & $70.2(2)$ & $C(3)$ & $C(4)$ & $C(5)$ & $107.4(3)$ \\
\hline $\mathrm{Fe}(1)$ & $C(5)$ & $c(1)$ & $70.8(2)$ & $\mathrm{Fe}(1)$ & $C(5)$ & $C(4)$ & $70.2(2)$ \\
\hline$C(1)$ & $C(5)$ & $C(4)$ & $108.9(3)$ & $\mathrm{Fe}(1)$ & $c(6)$ & $N(1)$ & $172.7(3)$ \\
\hline$N(3)$ & $c(7)$ & $C(8)$ & $104.8(2)$ & $N(2)$ & $C(8)$ & $c(7)$ & $104.2(2)$ \\
\hline$N(5)$ & $C(12)$ & $c(13)$ & $105.3(2)$ & $N(4)$ & $c(13)$ & $c(12)$ & $105.0(2)$ \\
\hline
\end{tabular}


Table 6. Bond Angles( $\left.{ }^{\circ}\right)$

\begin{tabular}{|c|c|c|c|c|c|c|c|}
\hline atom & atom & atom & angle & atom & atom & atom & angle \\
\hline $\mathrm{Fe}(1)$ & $c(1)$ & $\mathrm{H}(1)$ & 126.0 & $C(2)$ & $c(1)$ & $\mathrm{H}(1)$ & 135.7 \\
\hline$C(5)$ & $c(1)$ & $\mathrm{H}(1)$ & 117.1 & $\mathrm{Fe}(1)$ & $C(2)$ & $\mathrm{H}(2)$ & 119.0 \\
\hline$C(1)$ & $c(2)$. & $\mathrm{H}(2)$ & 129.7 & $c(3)$ & $c(2)$ & $\mathrm{H}(2)$ & 121.7 \\
\hline $\mathrm{Fe}(1)$ & $C(3)$ & $\mathrm{H}(3)$ & 114.7 & $C(2)$ & $c(3)$ & $\mathrm{H}(3)$ & 110.8 \\
\hline$C(4)$ & $c(3)$ & $\mathrm{H}(3)$ & 139.5 & $\mathrm{Fe}(1)$ & $C(4)$ & $\mathrm{H}(4)$ & 116.6 \\
\hline$c(3)$ & $C(4)$ & $\mathrm{H}(4)$ & 121.6 & $c(5)$ & $C(4)$ & $\mathrm{H}(4)$ & 130.1 \\
\hline $\mathrm{Fe}(1)$ & $C(5)$ & $\mathrm{H}(5)$ & 108.6 & $c(1)$ & $C(5)$ & $\mathrm{H}(5)$ & 118.7 \\
\hline$C(4)$ & $c(5)$ & $\mathrm{H}(5)$ & 129.1 & $N(3)$ & $c(7)$ & $\mathrm{H}(6)$ & 111.8 \\
\hline$N(3)$ & $c(7)$ & $\mathrm{H}(7)$ & 110.4 & $c(8)$ & $c(7)$ & $\mathrm{H}(6)$ & 110.3 \\
\hline$C(8)$ & $C(7)$ & $\mathrm{H}(7)$ & 107.2 & $\mathrm{H}(6)$ & $C(7)$ & $\mathrm{H}(7)$ & 112.0 \\
\hline$N(2)$ & $C(8)$ & $\mathrm{H}(8)$ & 105.7 & $N(2)$ & $c(8)$ & $\mathrm{H}(9)$ & 106.1 \\
\hline$c(7)$ & $c(8)$ & $\mathrm{H}(8)$ & 109.5 & $c(7)$ & $C(8)$ & $\mathrm{H}(9)$ & 113.2 \\
\hline $\mathrm{H}(8)$ & $C(8)$ & $\mathrm{H}(9)$ & 117.1 & $N(3)$ & $C(9)$ & $\mathrm{H}(10)$ & 112.4 \\
\hline$N(3)$ & $C(9)$ & $\mathrm{H}(11)$ & 113.2 & $N(3)$ & $c(9)$ & $\mathrm{H}(12)$ & 111.2 \\
\hline $\mathrm{H}(10)$ & $c(9)$ & $\mathrm{H}(11)$ & 118.3 & $\mathrm{H}(10)$ & $c(9)$ & $\mathrm{H}(12)$ & 110.9 \\
\hline $\mathrm{H}(11)$ & $c(9)$ & $\mathrm{H}(12)$ & 88.5 & $\mathrm{~N}(2)$ & $C(10)$ & $\mathrm{H}(13)$ & 102.2 \\
\hline $\mathrm{N}(2)$ & $c(10)$ & $\mathrm{H}(14)$ & 109.6 & $N(2)$ & $C(10)$ & $\mathrm{H}(15)$ & 122.7 \\
\hline H $(13)$ & $C(10)$ & $\mathrm{B}(14)$ & 122.1 & $\mathrm{H}(13)$ & $c(10)$ & $\mathrm{H}(15)$ & 86.9 \\
\hline $\mathrm{H}(14)$ & $c(10)$ & $\mathrm{H}(15)$ & 112.3 & $0(1)$ & $C(11)$ & $\mathrm{H}(16)$ & 114.7 \\
\hline $0(1)$ & $c(11)$ & $\mathrm{H}(17)$ & 108.9 & $0(1)$ & $C(11)$ & $\mathrm{H}(18)$ & 109.4 \\
\hline $\mathrm{H}(16)$ & $C(11)$ & $\mathrm{H}(17)$ & 106.8 & $\mathrm{H}(16)$ & $C(11)$ & $\mathrm{H}(18)$ & 107.7 \\
\hline $\mathrm{H}(17)$ & $C(11)$ & $\mathrm{H}(18)$ & 109.2 & $N(5)$ & $C(12)$ & $\mathrm{H}(19)$ & 111.0 \\
\hline $\mathrm{N}(5)$ & $C(12)$ & $\mathrm{H}(20)$ & 106.4 & $c(13)$ & $c(12)$ & $\mathrm{H}(19)$ & 115.1 \\
\hline$C(13)$ & $C(12)$ & $\mathrm{H}(20)$ & 113.7 & $\mathrm{H}(19)$ & $C(12)$ & $\mathrm{H}(20)$ & 105.0 \\
\hline $\mathrm{N}(4)$ & $c(13)$ & $\mathrm{B}(21)$ & 106.8 & $N(4)$ & $c(13)$ & $\mathrm{H}(22)$ & 109.4 \\
\hline$C(12)$ & $c(13)$ & $\mathrm{H}(21)$ & 113.6 & $c(12)$ & $c(13)$ & $\mathrm{H}(22)$ & 11.5 .5 \\
\hline $\mathrm{H}(21)$ & $c(13)$ & $\mathrm{H}(22)$ & 106.2 & $N(5)$ & $c(14)$ & $\mathrm{H}(23)$ & 105.2 \\
\hline$N(5)$ & $C(14)$ & $\mathrm{H}(24)$ & 108.5 & $\mathrm{~N}(5)$ & $C(14)$ & $\mathrm{H}(25)$ & 107.7 \\
\hline $\mathrm{H}(23)$ & $C(14)$ & $\mathrm{H}(24)$ & 111.4 & $\mathrm{H}(23)$ & $C(14)$ & $\mathrm{H}(25)$ & 114.9 \\
\hline $\mathrm{H}(24)$ & $C(14)$ & $\mathrm{H}(25)$ & 108.9 & $\mathrm{~N}(4)$ & $C(15)$ & $\mathrm{H}(26)$ & 112.0 \\
\hline $\mathrm{N}(4)$ & $c(15)$ & $\mathrm{H}(27)$ & 119.2 & $N(4)$ & $c(15)$ & $\mathrm{H}(28)$ & 114.2 \\
\hline $\mathrm{H}(26)$ & $c(15)$ & $\mathrm{H}(27)$ & 103.6 & $\mathrm{H}(26)$ & $c(15)$ & $\mathrm{H}(28)$ & 102.1 \\
\hline $\mathrm{H}(27)$ & $C(15)$ & $\mathrm{H}(28)$ & 103.8 & $0(2)$ & $c(16)$ & H(29) & 99.1 \\
\hline $0(2)$ & $C(16)$ & $\mathrm{H}(30)$ & 113.8 & $0(2)$ & $c(16)$ & $\mathrm{H}(31)$ & 102.9 \\
\hline $\mathrm{H}(29)$ & $c(16)$ & $\mathrm{H}(30)$ & 113.7 & H(29) & $c(16)$ & $\mathrm{H}(31)$ & 109.2 \\
\hline
\end{tabular}


Table 7. Torsion Angles( $\left.{ }^{\circ}\right)$

\begin{tabular}{|c|c|c|c|c|c|c|c|c|c|}
\hline & & & & & & & & & \\
\hline (1) & $P(1)$ & (1) & (11) & $179.3(2)$ & $(1)$ & $P(1)$ & (2) & $c(8)$ & \\
\hline 1 & & & & & & & & & \\
\hline (1) & $P(1)$ & $N(3)$ & (9) & -70.5 & & & 2) & & (3) \\
\hline & & & & -143 & & & & & \\
\hline & 2) & & & & & & & & \\
\hline & & & & & & & & & \\
\hline & & & & & & & & & \\
\hline & & & & -60. & & & & & \\
\hline & & & & -60. & & & & & \\
\hline (1) & (5) & $c(1)$ & $r$ & 59. & & & & & \\
\hline 1) & & & & 39. & & & 2) & & \\
\hline & & & & & & & 1) & & \\
\hline & & & & -15 & & & 2) & & \\
\hline & & & & 0 & & & & & \\
\hline & & & & -17 & & & & & \\
\hline & & & & 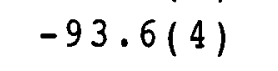 & & & & & \\
\hline & & ci & & & & & & & \\
\hline & & $c(8)$ & & -3 & & & & & \\
\hline & & & & & & & & & \\
\hline & & & & -7 & & & & & \\
\hline & & & & & & & & & \\
\hline & & & & 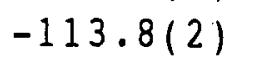 & & & & & \\
\hline & & & & & & & & & \\
\hline & & & & & & & & & \\
\hline & & & cl & 9 & & & & & \\
\hline & & Cl & & & & & & & \\
\hline & $P(1)$ & Fel & & & & & & & \\
\hline & & el & & & & & & & \\
\hline 1) & $\mathrm{P}$ & 1 & & -9 & & & & & \\
\hline & & No & & & & & & & \\
\hline & & & & & & & & $c(9)$ & \\
\hline & & & & & & & e(1) & $c(2)$ & -14 \\
\hline & & & & & & $P(2)$ & $\mathrm{Fe}(1)$ & & \\
\hline & & & & 176.6 & & & & & \\
\hline & & & & 91.1 & & & & & \\
\hline & 121 & ) & & $114.5(2)$ & $0(2)$ & $P(2)$ & $N(5)$ & $C(14)$ & (2) \\
\hline
\end{tabular}


Table 7. Torsion Angles $\left(^{\circ}\right)$ (continued)

\begin{tabular}{|c|c|c|c|c|c|c|c|c|c|}
\hline atom & om & & & & & & & atom & angle \\
\hline$N(1)$ & $c(6)$ & $\mathrm{Fe}(1)$ & $c(1)$ & $-26(1)$ & (1) & $c(6)$ & (1) & $c(2)$ & $-51(1)$ \\
\hline $\mathrm{N}(1)$ & $c(6)$ & $\mathrm{Fe}(1)$ & $c(3)$ & $-24(1)$ & (1) & $c(6)$ & $\mathrm{Fe}(1)$ & $c(4)$ & $14(1)$ \\
\hline$N(1)$ & $c(6)$ & $\mathrm{Fe}(1)$ & $c(5)$ & $29(1)$ & (2) & $P(1)$ & $\mathrm{Fe}(1)$ & $C(1)$ & 157.21 \\
\hline $\mathrm{N}(2)$ & $P(1)$ & $e(1)$ & $c(2)$ & -163 & $\mathrm{~N}(2)$ & $P(1)$ & $\mathrm{Fe}(1)$ & $c(3)$ & -133.6 \\
\hline$N(2)$ & $P(1)$ & (1) & $c(4)$ & -151.5 & $N(2)$ & $P(1)$ & $\mathrm{Fe}(1)$ & $c(5)$ & 138.2 \\
\hline (2) & (1) & $\mathrm{Fe}(1)$ & $c(6)$ & $-46.2(1)$ & $N(2)$ & $P(1)$ & $0(1)$ & $c(11)$ & -44 \\
\hline (2) & (1) & $N(3)$ & $C(7)$ & $7.2(2)$ & $N(2)$ & $P(1)$ & $N(3)$ & $c(9)$ & 155.412 \\
\hline (2) & $c(8)$ & $c(7)$ & $N(3)$ & $35.5(3)$ & $N(3)$ & $P(1)$ & $\mathrm{Fe}(1)$ & $c(1)$ & $41.0(1)$ \\
\hline$N(3)$ & $P(1)$ & $F e(1)$ & $c(2)$ & 80.5 & $N(3)$ & $P(1)$ & $\mathrm{Fe}(1)$ & $c(3)$ & $110.2(1)$ \\
\hline$N(3)$ & $P(1)$ & $\mathrm{Fe}(1)$ & $c(4)$ & $92.2(3)$ & (3) & $P(1)$ & $F e(1)$ & $c(5)$ & $22.0(2)$ \\
\hline$N(3)$ & $P(1)$ & $\mathrm{Fe}(1)$ & $c(6)$ & $-162.4(1)$ & (3) & $P(1)$ & $0(1)$ & $c(11)$ & $51.1(2)$ \\
\hline$N(3)$ & $P(1)$ & $N(2)$ & $\mathrm{C}(8)$ & 16.2 & (3) & $P(1)$ & $N(2)$ & $c(10)$ & $170.8(2)$ \\
\hline $\mathrm{N}(4)$ & $P(2)$ & $e(1)$ & $C(1)$ & 93.7 & $N(4)$ & $P(2)$ & $\mathrm{Fe}(1)$ & $c(2)$ & $(2)$ \\
\hline $\mathbb{N}(4)$ & $P(2)$ & $e(1)$ & $c(3)$ & 16.4 & $\mathrm{~N}(4)$ & $P(2)$ & $\mathrm{Fe}($ & c & ( 2 \\
\hline$N(4)$ & $P(2)$ & $\mathrm{Fe}(1)$ & $c(5)$ & 60. & $\mathrm{~N}(4)$ & $P(2)$ & & $\mathrm{c}$ & $-70.3(1$ \\
\hline$N(4)$ & $P(2)$ & $0(2)$ & $c(16)$ & -52.5 & $N(4)$ & $P(2)$ & $N(5)$ & $\mathrm{c}($ & -6 \\
\hline$N(4)$ & $P(2)$ & $N(5)$ & $C(14)$ & -150.2 & $\mathrm{~N}(4)$ & $c(13)$ & $c(12)$ & $N(5)$ & -33 \\
\hline 51 & $P(2)$ & $e(1)$ & $C(1)$ & -18.2 & $N(5)$ & $P(2)$ & $\mathrm{Fe}$ & $C(2)$ & -21 \\
\hline 5) & $P(2)$ & (1) & $c(3)$ & -95. & $\mathrm{~N}$ & $\mathrm{P}$ & $\mathrm{Fe}$ & $\mathrm{c}$ & -87. \\
\hline 5) & $P(2)$ & $F \in(1)$ & $c(5)$ & -51 & ) & $P(2)$ & $\mathrm{Fe}(1)$ & c & (1) \\
\hline (5) & $P(2)$ & $0(2)$ & $c(16)$ & 44. & & $P(2)$ & $N(4)$ & 3) & (3) \\
\hline$N(5)$ & $P(2)$ & $N(4)$ & $C(15)$ & -163 & (1) & $\mathrm{Fe}(1)$ & $c(2)$ & $c(3)$ & $-118.4(3)$ \\
\hline$c(1)$ & $\mathrm{Fe}$ & $c(3)$ & $c(2)$ & 38. & ) & $\mathrm{Fe}(1)$ & $c(3)$ & $c(4)$ & $-81.2(2)$ \\
\hline$c(1)$ & $\mathrm{Fel}$ & $c(4)$ & $c(3)$ & 80. & (1) & $\mathrm{Fe}(1)$ & $c(4)$ & $c(5)$ & $-37.2(2)$ \\
\hline$c(1)$ & $\mathrm{Fe}(1)$ & $c(5)$ & $c(4)$ & 119.3 & (1) & $c(2)$ & $\mathrm{Fe}(1)$ & $c(3)$ & $118.4(3)$ \\
\hline$c(1)$ & $c(2)$ & $\mathrm{Fe}(1)$ & $C(4)$ & 80. & (1) & $c(2)$ & $\mathrm{Fe}(1)$ & $c(5)$ & $37.6(2)$ \\
\hline$c(1)$ & $c(2)$ & $\mathrm{Fe}(1)$ & $c(6)$ & 163. & $c(1)$ & $c(2)$ & $c(3)$ & $c(4)$ & (3) \\
\hline$C(1)$ & $C(5)$ & $F e(1)$ & $c(2)$ & -38.0 & $c(1)$ & $c(5)$ & $\mathrm{Fe}(1)$ & $c(3)$ & -81 . \\
\hline$c(1)$ & $c(5)$ & $\mathrm{Fe}(1)$ & $c(4)$ & -119.3 & $c(1)$ & $c(5)$ & $\mathrm{Fe}(1)$ & $c(6)$ & -144. \\
\hline$c(1)$ & (5) & $C(4)$ & $c(3)$ & $-0.1(3)$ & $c(2)$ & $\mathrm{Fe}(1)$ & $c(1)$ & $c(5)$ & $118.3(3)$ \\
\hline$c(2)$ & 1) & $c(3)$ & $c(4)$ & $119.1(3)$ & Cl & $\mathrm{Fe}$ & ci & $c(3)$ & $37.1(2)$ \\
\hline$c(2)$ & (1) & $C(4)$ & $c(5)$ & $-80.8(2)$ & $\mathrm{C}(2$ & Fe (1) & $c(5)$ & $c(4)$ & $81.2(2)$ \\
\hline$c(2)$ & & $F e(1)$ & $c(3)$ & $-37.5(2)$ & $c($ & $c(1)$ & $\mathrm{Fe}(1)$ & $c(4)$ & $-81.0(2)$ \\
\hline$f(2$ & 1) & $F e(1)$ & $c(5)$ & $-118.3(3)$ & & $c(1)$ & $\mathrm{Fe}(1)$ & $c(6)$ & $-35.5(4)$ \\
\hline 12 & 1) & $c(5)$ & $\mathrm{C}(4)$ & -0.4 & & $c(3)$ & $\mathrm{Fe}(1) \mathrm{C}$ & $C(4)$ & $119.1(3)$ \\
\hline (2) & (3) & $e(1)$ & $c(5)$ & $81.2(2)$ & $c(2)$. & $c(3)$ & $\mathrm{Fe}(1) \mathrm{C}$ & $C(6)$ & $-141.2(2)$ \\
\hline
\end{tabular}




\begin{tabular}{|c|c|c|c|c|c|c|c|c|c|}
\hline tom & atom & atom & atom & angle & atom & atom & atom & a tom & angle \\
\hline (2) & $c(3)$ & $C(4)$ & $c(5)$ & $0.7(3)$ & $c(3)$ & $\mathrm{Fe}(1)$ & $c(1)$ & $c(5)$ & 80.812 \\
\hline (3) & $\mathrm{Fe}(1)$ & $C(4)$ & $c(5)$ & $-117.9(3)$ & $c(3)$ & $\mathrm{Fe}(1)$ & $c(5)$ & $c(4)$ & $38.1(2)$ \\
\hline (3) & $c(2)$ & $\mathrm{Fe}(1)$ & $\mathrm{C}(4)$ & $-37.5(2)$. & $c(3)$ & $c(2)$ & $\mathrm{Fe}(1)$ & $c(5)$ & $-80.8(2)$ \\
\hline (3) & $c(2)$ & $\dot{\mathrm{F}} \mathrm{e}(1)$ & $c(6)$ & $44.6(2)$ & $C(3)$ & $\mathrm{C}(2)$ & $C(1)$ & $C(5)$ & $0.8(3)$ \\
\hline$c(3)$ & $C(4)$ & $\mathrm{Fe}(1)$ & $c(5)$ & $117.9(3)$ & $c(3)$ & $C(4)$ & Fe(1) & $c(6)$ & $-80.6(2)$ \\
\hline$c(4)$ & $F \in(1)$ & $c(1)$ & $c(5)$ & $37.3(2)$ & $C(4)$ & $c(3)$ & $F e(1)$ & $C(5)$ & $-37.9(2)$ \\
\hline$c(4)$ & $c(3)$ & $\mathrm{Fe}(1)$ & $c(6)$ & $99.7(2)$ & $C(4)$ & $c(5)$ & $F \in(1)$ & $c(6)$ & $-24.7(3)$ \\
\hline$c(5)$ & $C(1)$ & $\mathrm{Fe}(1)$ & $c(6)$ & $82.8(4)$ & $c(5)$ & $c(4)$ & $\mathrm{Fe}(1)$ & $c(6)$ & $161.5(2)$ \\
\hline ( 7 ) & $c(8)$ & $\mathrm{N}(2)$ & $C(10)$ & $170.2(2)$ & $c(8)$ & $c(7)$ & $\mathrm{N}(3)$ & $c(9)$ & $-176.6(3)$ \\
\hline (12) & $c(13)$ & $N(4)$ & $c(15)$ & $-178 \cdot 7(3)$ & $c(13)$ & $c(12)$ & $N(5)$ & $C(14)$ & $171.4(2)$ \\
\hline
\end{tabular}

Table 8. Non-bonded Contacts out to $3.60 \AA$

$\begin{array}{llllll}\text { atom } & \text { atom } & \text { distance } & \text { atom } & \text { atom } & \text { distance } \\ 0(1) & \mathrm{C}(9)^{1)} & 3.350(4) & \mathrm{N}(1) & \mathrm{C}(12)^{2)} & 3.540(4) \\ \mathrm{C}(4) & \mathrm{C}(14)^{3)} & 3.542(4) & \mathrm{C}(11) & \left.\mathrm{C}(16)^{4}\right) & 3.536(5)\end{array}$

Symmetry operations

(1) $\quad X,-Y, Z+1 / 2$

(3) $X-1 / 2,-Y+1 / 2, Z+1 / 2$

$\mathrm{X}+1 / 2,-\mathrm{Y}+1 / 2, \mathrm{Z}+1 / 2$

$\mathrm{X}-1 / 2, \mathrm{Y}-1 / 2, \mathrm{Z}$ 


\title{
X-ray Structure Reports for Complex $\mathbf{3 0}$
}

\author{
A. Crystal Data
}

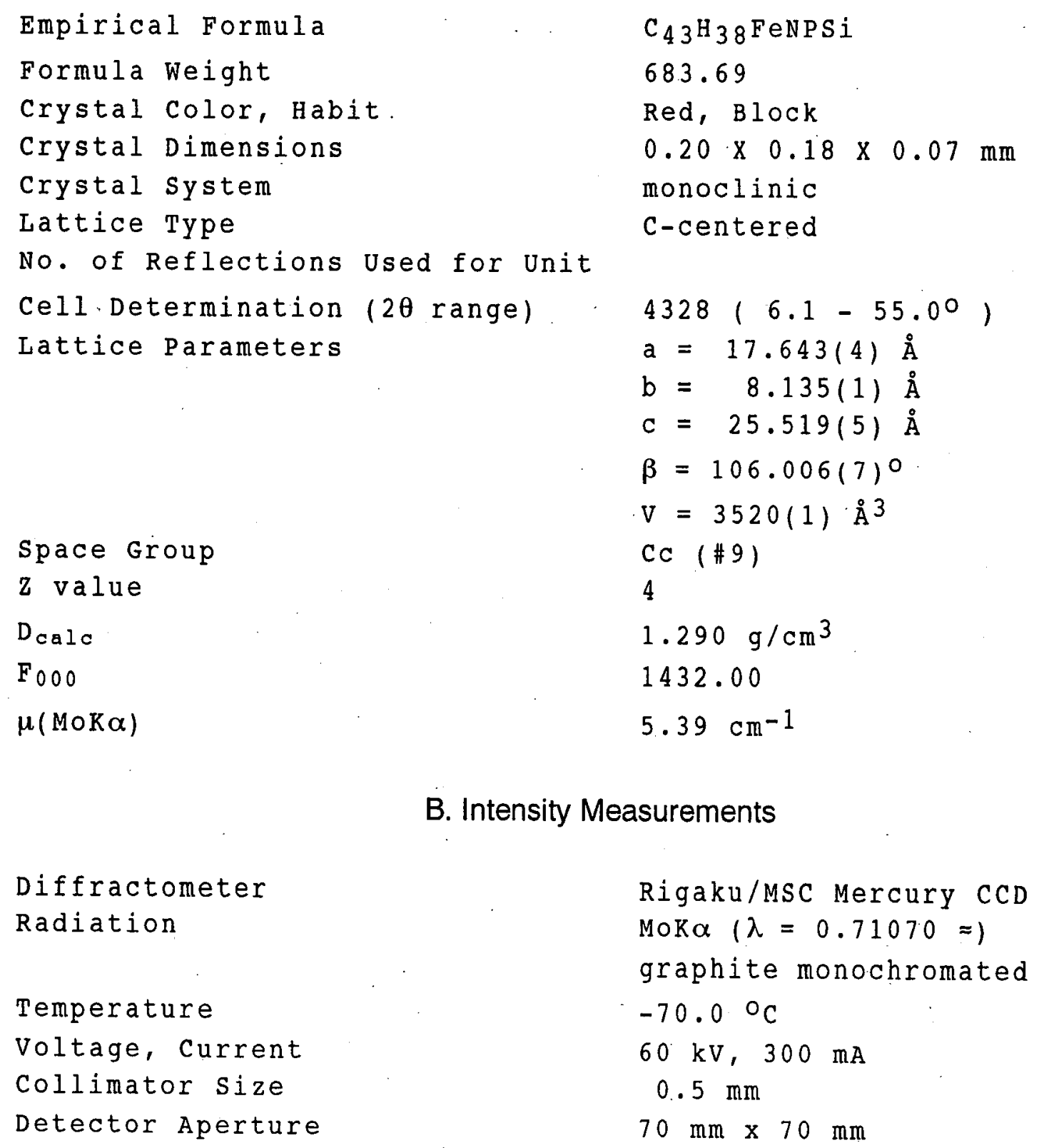




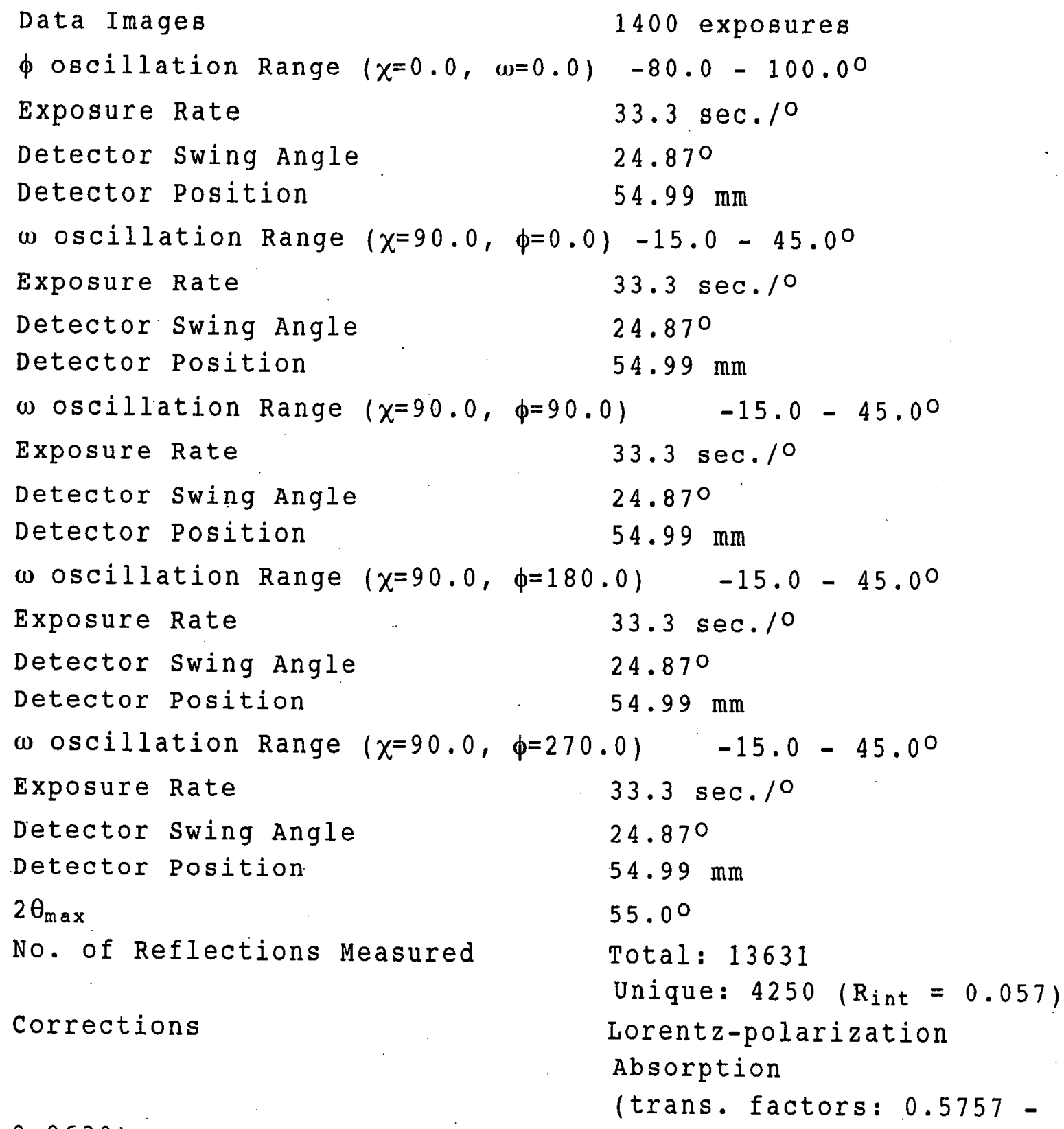

$0.9630)$

C. Structure Solution and Refinement

Structure solution

Refinement

Function Minimized

Least Squares weights
Direct Methods (SIR92)

Full-matrix least-squares

$\sum \mathrm{w}(|\mathrm{FO}|-|\mathrm{FC}|)^{2}$

$1 / \mathrm{\sigma}^{2}(\mathrm{FO})=4 \mathrm{FO}^{2} / \mathrm{\sigma}^{2}\left(\mathrm{FO}^{2}\right)$ 


\begin{tabular}{|c|c|}
\hline$p-f a c t o r$ & 0.0400 \\
\hline Anomalous Dispersion & All non-hydrogen a toms \\
\hline No. of observations ( $I>2.00 \sigma(I)$, & $2 \theta<54.97^{\circ}, 5567$ \\
\hline No. Variables & 424 \\
\hline Reflection/Parameter Ratio & 13.13 \\
\hline Residuals: $R$; $R w$ & $0.067 ; 0.074$ \\
\hline Residuals: R1 & 0.067 \\
\hline No. of Reflections to calc R1 & 5567 \\
\hline Goodness of Fit Indicator & 1.61 \\
\hline Max Shift/Error in Final cycle & 0.000 \\
\hline Maximum peak in Final Diff. Map & $0.43 \mathrm{e}^{-/ \AA^{3}}$ \\
\hline Minimum peak in Final Diff. Map & $-0.56 e^{-/ / \AA^{3}}$ \\
\hline
\end{tabular}


Table 9. Atomic coordinates and $B_{i s o} / B_{\theta q}$

\begin{tabular}{|c|c|c|c|c|}
\hline atom & $\mathrm{x}$ & y & 2 & $B_{\theta q}$ \\
\hline $\mathrm{Fe}(1)$ & $0.5554(6)$ & $-0.46139(7)$ & $0.4985(4)$ & $2.28(1)$ \\
\hline$P(1)$ & $0.5868(6)$ & $-0.3202(1)$ & $0.5741(4)$ & $2.25(2)$ \\
\hline $\operatorname{Si}(1)$ & $0.5080(6)$ & $-0.0881(1)$ & $0.3617(4)$ & $2.44(2)$ \\
\hline $\mathrm{N}(1)$ & $0.5373(6)$ & $-0.1801(5)$ & $0.4239(4)$ & $3.37(9)$ \\
\hline$C(1)$ & $0.5434(6)$ & $-0.2873(5)$ & $0.4560(4)$ & $2.07(8)$ \\
\hline$c(2)$ & $0.4379(6)$ & $-0.4601(6)$ & $0.4944(5)$ & $3.5(1)$ \\
\hline$c(3)$ & $0.5355(6)$ & $-0.7091(6)$ & $0.4740(5)$ & $3.6(1)$ \\
\hline$C(4)$ & $0.5631(7)$ & $-0.6232(6)$ & $0.4369(5)$ & $3.9(1)$ \\
\hline$c(5)$ & $0.6407(7)$ & $-0.5608(6)$ & $0.4650(5)$ & $4.2(1)$ \\
\hline$c(6)$ & $0.6572(6)$ & $-0.6068(6)$ & $0.5198(5)$ & $4.1(1)$ \\
\hline$C(7)$ & $0.5915(7)$ & $-0.7040(6)$ & $0.5255(5)$ & $3.6(1)$ \\
\hline$c(8)$ & $0.6198(6)$ & $-0.4504(5)$ & $0.6355(5)$ & $2.52(8)$ \\
\hline$C(9)$ & $0.5680(6)$ & $-0.5728(6)$ & $0.6428(5)$ & $3.1(1)$ \\
\hline$c(10)$ & $0.5905(7)$ & $-0.6846(6)$ & $0.6856(5)$ & $3.9(1)$ \\
\hline$c(11)$ & $0.6653(7)$ & $-0.6786(6)$ & $0.7214(5)$ & $4.0(1)$ \\
\hline$C(12)$ & $0.7157(7)$ & $-0.5576(6)$ & $0.7140(5)$ & $4.0(1)$ \\
\hline$c(13)$ & $0.6942(6)$ & $-0.4458(6)$ & $0.6707(5)$ & $3.35(10)$ \\
\hline$C(14)$ & $0.6667(6)$ & $-0.1702(5)$ & $0.5813(5)$ & $2.47(8)$ \\
\hline$C(15)$ & $0.7122(6)$ & $-0.1769(6)$ & $0.5439(5)$ & $3.4(1)$ \\
\hline$c(16)$ & $0.7717(6)$ & $-0.0624(7)$ & $0.5464(5)$ & $3.9(1)$ \\
\hline$c(17)$ & $0.7853(7)$ & $0.0599(6)$ & $0.5842(5)$ & $4.1(1)$ \\
\hline$C(18)$ & $0.7409(7)$ & $0.0708(6)$ & $0.6216(5)$ & $3.8(1)$ \\
\hline$C(19)$ & $0.6815(6)$ & $-0.0440(6)$ & $0.6194(5)$ & $2.93(10)$ \\
\hline$c(20)$ & $0.5123(6)$ & $-0.1856(5)$ & $0.5905(5)$ & $2.40(8)$ \\
\hline$c(21)$ & $0.4927(6)$ & $-0.1896(6)$ & $0.6398(5)$ & $3.1(1)$ \\
\hline$C(22)$ & $0.4370(7)$ & $-0.0818(7)$ & $0.6494(5)$ & $3.8(1)$ \\
\hline$c(23)$ & $0.4019(6)$ & $0.0332(7)$ & $0.6105(5)$ & $4.0(1)$ \\
\hline$C(24)$ & $0.4215(7)$ & $0.0384(7)$ & $0.5626(5)$ & $4.2(1)$ \\
\hline$C(25)$ & $0.4764(6)$ & $-0.0682(6)$ & $0.5518(5)$ & $3.6(1)$ \\
\hline$C(26)$ & $0.5940(6)$ & $0.0228(5)$ & $0.3501(5)$ & $2.54(8)$ \\
\hline$C(27)$ & $0.6542(6)$ & $0.0819(6)$ & $0.3935(5)$ & $3.4(1)$ \\
\hline$c(28)$ & $0.7188(6)$ & $0.1668(7)$ & $0.3844(5)$ & $4.3(1)$ \\
\hline$C(29)$ & $0.7210(6)$ & $0.1941(7)$ & $0.3318(5)$ & $4.8(1)$ \\
\hline$c(30)$ & $0.6629(7)$ & $0.1393(7)$ & $0.2879(.5)$ & $4.4(1)$ \\
\hline$c(31)$ & $0.6002(6)$ & $0.0514(6)$ & $0.2979(5)$ & $3.30(10)$ \\
\hline$c(32)$ & $0.4758(6)$ & $-0.2481(5)$ & $0.3080(5)$ & $2.54(8)$ \\
\hline
\end{tabular}


Table 9. Atomic coordinates and Biso/Bog (continued)

\begin{tabular}{|c|c|c|c|c|}
\hline atom & $\mathrm{x}$ & $\mathrm{y}$ & $z$ & $B_{\theta q}$ \\
\hline$c(33)$ & $0.5245(6)$ & $-0.3825(5)$ & $0.3059(5)$ & $2.93(10)$ \\
\hline$C(34)$ & $0.5019(6)$ & $-0.5021(6)$ & $0.2665(5)$ & $3.3(1)$ \\
\hline$C(35)$ & $0.4300(7)$ & $-0.4922(6)$ & $0.2283(5)$ & $3.6(1)$ \\
\hline$c(36)$ & $0.3801(7)$ & $-0.3611(7)$ & $0.2293(5)$ & $4.4(1)$ \\
\hline$c(37)$ & $0.4027(6)$ & $-0.2408(6)$ & $0.2688(5)$ & $3.7(1)$ \\
\hline$c(38)$ & $0.4244(6)$ & $0.0510(6)$ & $0.3622(5)$ & $2.75(9)$ \\
\hline$C(39)$ & $0.4089(6)$ & $0.1935(6)$ & $0.3323(5)$ & $3.5(1)$ \\
\hline$C(40)$ & $0.3468(6)$ & $0.2974(6)$ & $0.3329(5)$ & $4.7(1)$ \\
\hline$c(41)$ & $0.2998(6)$ & $0.2555(8)$ & $0.3660(5)$ & $5.0(1)$ \\
\hline$C(42)$ & $0.3125(7)$ & $0.1168(8)$ & $0.3952(5)$ & $5.5(2)$ \\
\hline$C(43)$ & $0.3754(6)$ & $0.0123(7)$ & $0.3950(5)$ & $4.1(1)$ \\
\hline $\mathrm{H}(1)$ & 0.4244 & -0.3585 & 0.5076 & $4 \cdot 4$ \\
\hline $\mathrm{H}(2)$ & 0.4075 & -0.4731 & 0.4571 & 4.4 \\
\hline $\mathrm{H}(3)$ & 0.4264 & -0.5478 & 0.5152 & 4.4 \\
\hline $\mathrm{H}(4)$ & 0.4864 & -0.7640 & 0.4670 & $4 \cdot 4$ \\
\hline $\mathrm{H}(5)$ & 0.5369 & -0.6117 & 0.3976 & 5.0 \\
\hline $\mathrm{H}(6)$ & 0.6760 & -0.4993 & 0.4494 & 5.1 \\
\hline $\mathrm{H}(7)$ & 0.7048 & -0.5857 & 0.5481 & 5.1 \\
\hline $\mathrm{H}(8)$ & 0.5882 & -0.7534 & 0.5583 & 4.7 \\
\hline $\mathrm{B}(9)$ & 0.5163 & -0.5794 & 0.6174 & 4.0 \\
\hline $\mathrm{H}(10)$ & 0.5537 & -0.7653 & 0.6900 & 5.0 \\
\hline $\mathrm{H}(11)$ & 0.6826 & -0.7556 & 0.7500 & 4.9 \\
\hline $\mathrm{H}(12)$ & 0.7670 & -0.5498 & 0.7381 & 5.0 \\
\hline $\mathrm{H}(13)$ & 0.7318 & -0.3678 & 0.6653 & 4.4 \\
\hline $\mathrm{H}(14)$ & 0.7035 & -0.2603 & 0.5168 & $4 \cdot 3$ \\
\hline $\mathrm{H}(15)$ & 0.8026 & -0.0694 & 0.5213 & 5.0 \\
\hline $\mathrm{H}(16)$ & 0.8267 & 0.1370 & 0.5846 & 5.2 \\
\hline $\mathrm{H}(17)$ & 0.7513 & 0.1572 & 0.6472 & 4.6 \\
\hline $\mathrm{H}(18)$ & 0.6504 & -0.0352 & 0.6444 & 3.6 \\
\hline $\mathrm{H}(19)$ & 0.5175 & -0.2679 & 0.6671 & 3.9 \\
\hline $\mathrm{H}(20)$ & 0.4222 & -0.0872 & 0.6823 & 4.9 \\
\hline $\mathrm{H}(21)$ & 0.3649 & 0.1083 & 0.6169 & 5.0 \\
\hline $\mathrm{H}(22)$ & 0.3973 & 0.1195 & 0.5362 & 5.3 \\
\hline $\mathrm{H}(23)$ & 0.4897 & -0.0600 & 0.5178 & 4.5 \\
\hline $\mathrm{H}(24)$ & 0.6519 & 0.0646 & 0.4296 & 4.2 \\
\hline $\mathrm{H}(25)$ & 0.7603 & 0.2040 & 0.4141 & 5.3 \\
\hline
\end{tabular}


(C) 2003 American Chemical Society, Organometallics, Nakazawa om0208319 Supporting İnfo Page 19

Table 9. Atomic coordinates and $B_{i s o} / B_{\theta q}$ (continued)

$\begin{array}{lcccc}\text { atom } & \mathrm{x} & \mathrm{y} & \mathrm{z} & \mathrm{B}_{\mathrm{\theta q}} \\ \mathrm{H}(26) & 0.7649 & 0.2532 & 0.3254 & 5.9 \\ \mathrm{H}(27) & 0.6646 & 0.1586 & 0.2517 & 5.7 \\ \mathrm{H}(28) & 0.5605 & 0.0114 & 0.2672 & 4.2 \\ \mathrm{H}(29) & 0.5742 & -0.3919 & 0.3321 & 3.7 \\ \mathrm{H}(30) & 0.5369 & -0.5907 & 0.2647 & 4.2 \\ \mathrm{H}(31) & 0.4139 & -0.5763 & 0.2011 & 4.6 \\ \mathrm{H}(32) & 0.3298 & -0.3511 & 0.2021 & 5.5 \\ \mathrm{H}(33) & 0.3676 & -0.1510 & 0.2688 & 4.7 \\ \mathrm{H}(34) & 0.4433 & 0.2219 & 0.3098 & 4.3 \\ \mathrm{H}(35) & 0.3372 & 0.3963 & 0.3105 & 5.9 \\ \mathrm{H}(36) & 0.2569 & 0.3253 & 0.3667 & 6.2 \\ \mathrm{H}(37) & 0.2788 & 0.0888 & 0.4165 & 6.8 \\ \mathrm{H}(38) & 0.3851 & -0.0851 & 0.4164 & 5.2 \\ & 0 & \end{array}$


(C) 2003 American Chemical Society, Organometallics, Nakazawa om0208319 Supporting Info Page 20

Table 10. Anisotropic Displacement Parameters

\begin{tabular}{|c|c|c|c|c|c|c|}
\hline tom & $\mathrm{U}_{11}$ & $\mathrm{U}_{22}$ & $\mathrm{U}_{33}$ & $\mathrm{U}_{12}$ & $\mathrm{U}_{13}$ & $\mathrm{U}_{23}$ \\
\hline Fe(1) & $0.0288(3)$ & $0.0278(3)$ & $0.0325(4)$ & $-0.0015(3)$ & $0.0122(2)$ & 0.000413 \\
\hline$P(1)$ & $0.0261(5)$ & $0.0306(5)$ & $0.0318(7)$ & $-0.0009(4)$ & $0.0128(4)$ & $10.0035(5)$ \\
\hline Si(1) & $0.0346(6)$ & $0.0283(6)$ & $0.0318(7)$ & $-0.0013(4)$ & $0.0124(5)$ & $1-0.0014(5$ \\
\hline $\mathrm{N}(1)$ & $0.058(2)$ & $0.034(2)$ & $0.040(2)$ & $-0.001(2)$ & $0.022(2)$ & $0.007(2)$ \\
\hline$c(1)$ & $0.023(2)$ & $0.032(2)$ & $0.025(2)$ & $-0.001(1)$ & $0.008(2)$ & $-0.008(1)$ \\
\hline$c(2)$ & $0.026(2)$ & $0.052(3)$ & $0.056(4)$ & $-0.001(2)$ & $0.014(2)$ & $-0.001(3)$ \\
\hline$c(3)$ & $0.050(3)$ & $0.028(2)$ & $0.058(3)$ & $-0.008(2)$ & $0.016(2)$ & $-0.014(2)$ \\
\hline$c(4)$ & $0.069(3)$ & $0.041(3)$ & $0.041(3)$ & $0.001(2)$ & $0.018(2)$ & $-0.014(2)$ \\
\hline$c(5)$ & $0.057(2)$ & $0.041(3)$ & $0.079(4)$ & $0.009(2)$ & $0.050(2)$ & $-0.003(2)$ \\
\hline$c(6)$ & $0.038(2)$ & $0.044(3)$ & $0.072(3)$ & $0.009(2)$ & $0.013(3)$ & $-0.013(3)$ \\
\hline$c(7)$ & $0.064(3)$ & $0.027(2)$ & $0.049(3)$ & $0.009(2)$ & $0.020(2)$ & $0.010(2)$ \\
\hline$c(8)$ & $0.036(2)$ & $0.030(2)$ & $0.031(2)$ & $0.007(2)$ & $0.011(2)$ & $0.006(2)$ \\
\hline$c(9)$ & $0.045(3)$ & $0.039(2)$ & $0.032(3)$ & $-0.005(2)$ & $0.008(2)$ & $0.008(2)$ \\
\hline$c(10)$ & $0.069 .(3)$ & $0.044(3)$ & $0.043(3)$ & $-0: 001(2)$ & $0.027(2)$ & $0.013(2)$ \\
\hline$c(11)$ & $0.078(3)$ & $0.037(2)$ & $0.037(3)$ & $0.005(2)$ & $0.016(2)$ & $0.009(2)$ \\
\hline$c(12)$ & $0.056(3)$ & $0.051(3)$ & $0.035(3)$ & $0.000(2)$ & $-0.004(2)$ & $0.006(2)$ \\
\hline$c(13)$ & $0.039(2)$ & $0.036(2)$ & $0.047(3)$ & $-0: 001(2)$ & $0.003(2)$ & $0.005(2)$ \\
\hline$c(14)$ & $0.027(2)$ & $0.033(2)$ & $0.034(3)$ & $0.001(1)$ & $0.010(2)$ & $0.005(2)$ \\
\hline$C(15)$ & $0.031(2)$ & $0.054(3)$ & $0.051(3)$ & $-0.005(2)$ & $0.022(2)$ & $0.003(2)$ \\
\hline$c(16)$ & $0.032(2)$ & $0.064(3)$ & $0.055(4)$ & $-0.008(2)$ & $0.018(2)$ & $0.001(2)$ \\
\hline$c(17)$ & $0.040(3)$ & $0.039(3)$ & $0.073(4)$ & $-0.007(2)$ & $0.013(2)$ & $0.010(2)$ \\
\hline$c(18)$ & $0.043(3)$ & $0.034(2)$ & $0.057(4)$ & $-0.001(2)$ & $-0.004(2)$ & $0.004(2)$ \\
\hline$c(19)$ & $0.038(2)$ & $0.039(2)$ & $0.030(3)$ & $0.000(2)$ & $0.002(2)$ & $-0.003(2)$ \\
\hline$c(20)$ & $0.033(2)$ & $0.029(2)$ & $0.033(2)$ & $-0.003(1)$ & $0.017(2)$ & $-0.001(2)$ \\
\hline$c(21)$ & $0.041(2)$ & $0.047(3)$ & $.0 .035(3)$ & $0.001(2)$ & $0.018(2)$ & $0.005(2)$ \\
\hline$c(22)$ & $0.050(3)$ & $0.063(3)$ & $0.044(3)$ & $0.002(2)$ & $0.033(2)$ & $-0.008 .(2)$ \\
\hline$c(23)$ & $0.048(3)$ & $0.052(3)$ & $0.060(3)$ & $0.007(2)$ & $0.028(3)$ & $-0.014(2)$ \\
\hline$c(24)$ & $0.062(3)$ & $0.047(3)$ & $0.055(3)$ & $0.023(2)$ & $0.023(3)$ & $0.009(3)$ \\
\hline$c(25)$ & $0.061(3)$ & $0.049(3)$ & $0.034(3)$ & $0.016(2)$ & $0.025(2)$ & $0.008(2)$ \\
\hline$c(26)$ & $0.030(2)$ & $0.029(2)$ & $0.037(2)$ & $0.006(1)$ & $0.010(2)$ & $-0.001(2)$ \\
\hline$c(27)$ & $0.032(2)$ & $0.050(3)$ & $0.048(3)$ & $-0.001(2)$ & $0.013(2)$ & $-0.013(2)$ \\
\hline$c(28)$ & $0.036(2)$ & $0.054(3)$ & $0.079(4)$ & $-0.012(2)$ & $0.024(3)$ & $-0.024(3)$ \\
\hline$c(29)$ & $0.050(3)$ & $0.058(3)$ & $0.086(4)$ & $-0.018(3)$ & $0.039(3)$ & $-0.011(3)$ \\
\hline$c(30)$ & $0.055(3)$ & $0.056(3)$ & $0.069 .(4)$ & $-0.007(2)$ & $0.037(2)$ & $0.005(3)$ \\
\hline$c(31)$ & $0.045(2)$ & $0.041(3)$ & $0.041(3)$ & $0.001(2)$ & $0.015(2)$ & $0.003(2)$ \\
\hline$c(32)$ & $0.040(2)$ & $0.025(2)$ & $0.035(2)$ & $0.005(1)$ & $0.016(2)$ & $0.003(1)$ \\
\hline
\end{tabular}


(C) 2003 American Chemical Society, Organometallics, Nakazawa om0208319 Supporting Info Page 21

Table 10. Anisotropic Displacement Parameters (continued)

\begin{tabular}{lclllll} 
atom & $\mathrm{U}_{11}$ & $\mathrm{U}_{22}$ & $\mathrm{U}_{33}$ & \multicolumn{1}{l}{$\mathrm{U}_{12}$} & \multicolumn{1}{l}{$\mathrm{U}_{13}$} & \multicolumn{1}{c}{$\mathrm{U}_{23}$} \\
$\mathrm{C}(33)$ & $0.036(2)$ & $0.037(2)$ & $0.040(3)$ & $0.007(2)$ & $0.014(2)$ & $-0.003(2)$ \\
$\mathrm{C}(34)$ & $0.050(2)$ & $0.034(2)$ & $0.047(3)$ & $0.007(2)$ & $0.021(2)$ & $-0.005(2)$ \\
$\mathrm{C}(35)$ & $0.058(2)$ & $0.037(2)$ & $0.044(3)$ & $-0.006(2)$ & $0.019(2)$ & $-0.013(2)$ \\
$\mathrm{C}(36)$ & $0.053(3)$ & $0.048(3)$ & $0.059(4)$ & $0.006(2)$ & $0.003(3)$ & $-0.015(2)$ \\
$\mathrm{C}(37)$ & $0.040(2)$ & $0.044(3)$ & $0.055(3)$ & $0.004(2)$ & $0.009(2)$ & $-0.009(2)$ \\
$\mathrm{C}(38)$ & $0.033(2)$ & $0.038(2)$ & $0.034(3)$ & $-0.007(1)$ & $0.010(2)$ & $-0.013(2)$ \\
$\mathrm{C}(39)$ & $0.041(2)$ & $0.039(2)$ & $0.059(4)$ & $0.005(2)$ & $0.022(3)$ & $0.000(2)$ \\
$\mathrm{C}(40)$ & $0.048(3)$ & $0.040(3)$ & $0.087(5)$ & $0.012(2)$ & $0.013(3)$ & $0.001(3)$ \\
$\mathrm{C}(41)$ & $0.038(3)$ & $0.065(3)$ & $0.085(5)$ & $0.011(2)$ & $0.016(3)$ & $-0.024(3)$ \\
$\mathrm{C}(42)$ & $0.052(3)$ & $0.070(4)$ & $0.102(6)$ & $0.005(3)$ & $0.049(4)$ & $-0.009(3)$ \\
$\mathrm{C}(43)$ & $0.042(3)$ & $0.059(3)$ & $0.066(4)$ & $-0.012(2)$ & $0.037(3)$ & $-0.014(3)$
\end{tabular}

The general temperature factor expression:

$\exp \left(-2 P I^{2}\left(a * 2 U_{11} h^{2}+b * 2 U_{22} k^{2}+c * 2 U_{33} I^{2}+2 a * b * U_{12} h k+2 a * c * U_{13} h l+\right.\right.$ $\left.\left.2 b * C * U_{23} k 1\right)\right)$ 
Table 11. Bond Lengths ( $)^{-}$

$\begin{array}{llllll}\text { atom } & \text { atom } & \text { distance } & \text { atom } & \text { atom } & \text { distance } \\ \mathrm{Fe}(1) & \mathrm{P}(1) & 2.182(2) & \mathrm{Fe}(1) & \mathrm{C}(1) & 1.760(5) \\ \mathrm{Fe}(1) & \mathrm{C}(2) & 2.045(5) & \mathrm{Fe}(1) & \mathrm{C}(3) & 2.110(6) \\ \mathrm{Fe}(1) & \mathrm{C}(4) & 2.084(7) & \mathrm{Fe}(1) & \mathrm{C}(5) & 2.087(6) \\ \mathrm{Fe}(1) & \mathrm{C}(6) & 2.094(6) & \mathrm{Fe}(1) & \mathrm{C}(7) & 2.129(6) \\ \mathrm{P}(1) & \mathrm{C}(8) & 1.847(6) & \mathrm{P}(1) & \mathrm{C}(14) & 1.835(5) \\ \mathrm{P}(1) & \mathrm{C}(20) & 1.846(5) & \mathrm{S}(1) & \mathrm{N}(1) & 1.701(6) \\ \mathrm{Si}(1) & \mathrm{C}(26) & 1.857(6) & \mathrm{S}(1) & \mathrm{C}(32) & 1.862(6) \\ \mathrm{Si}(1) & \mathrm{C}(38) & 1.862(6) & \mathrm{N}(1) & \mathrm{C}(1) & 1.181(7) \\ \mathrm{C}(3) & \mathrm{C}(4) & 1.371(10) & \mathrm{C}(3) & \mathrm{C}(7) & 1.409(9) \\ \mathrm{C}(4) & \mathrm{C}(5) & 1.45(1) & \mathrm{C}(5) & \mathrm{C}(6) & 1.40(1) \\ \mathrm{C}(6) & \mathrm{C}(7) & 1.444(9) & \mathrm{C}(8) & \mathrm{C}(9) & 1.399(8) \\ \mathrm{C}(8) & \mathrm{C}(13) & 1.371(8) & \mathrm{C}(9) & \mathrm{C}(10) & 1.392(9) \\ \mathrm{C}(10) & \mathrm{C}(11) & 1.38(1) & \mathrm{C}(11) & \mathrm{C}(12) & 1.374(9) \\ \mathrm{C}(12) & \mathrm{C}(13) & 1.400(9) & \mathrm{C}(14) & \mathrm{C}(15) & 1.406(8) \\ \mathrm{C}(14) & \mathrm{C}(19) & 1.388(8) & \mathrm{C}(15) & \mathrm{C}(16) & 1.392(8) \\ \mathrm{C}(16) & \mathrm{C}(17) & 1.36(1) & \mathrm{C}(17) & \mathrm{C}(18) & 1.40(1) \\ \mathrm{C}(18) & \mathrm{C}(19) & 1.393(9) & \mathrm{C}(20) & \mathrm{C}(21) & 1.396(8) \\ \mathrm{C}(20) & \mathrm{C}(25) & 1.395(8) & \mathrm{C}(21) & \mathrm{C}(22) & 1.388(8) \\ \mathrm{C}(22) & \mathrm{C}(23) & 1.38(1) & \mathrm{C}(23) & \mathrm{C}(24) & 1.358(10) \\ \mathrm{C}(24) & \mathrm{C}(25) & 1.385(8) & \mathrm{C}(26) & \mathrm{C}(27) & 1.391(9) \\ \mathrm{C}(26) & \mathrm{C}(31) & 1.386(9) & \mathrm{C}(27) & \mathrm{C}(28) & 1.405(9) \\ \mathrm{C}(28) & \mathrm{C}(29) & 1.37(1) & \mathrm{C}(29) & \mathrm{C}(30) & 1.37(1) \\ \mathrm{C}(30) & \mathrm{C}(31) & 1.400(8) & \mathrm{C}(32) & \mathrm{C}(33) & 1.400(7) \\ \mathrm{C}(32) & \mathrm{C}(37) & 1.399(9) & \mathrm{C}(33) & \mathrm{C}(34) & 1.376(9) \\ \mathrm{C}(34) & \mathrm{C}(35) & 1.373(10) & \mathrm{C}(35) & \mathrm{C}(36) & 1.387(9) \\ \mathrm{C}(36) & \mathrm{C}(37) & 1.383(9) & \mathrm{C}(38) & \mathrm{C}(39) & 1.374(9) \\ \mathrm{C}(38) & \mathrm{C}(43) & 1.395(8) & \mathrm{C}(39) & \mathrm{C}(40) & 1.387(8) \\ \mathrm{C}(40) & \mathrm{C}(41) & 1.38(1) & \mathrm{C}(41) & \mathrm{C}(42) & 1.34(1) \\ \mathrm{C}(42) & \mathrm{C}(43) & 1.398(10) & \mathrm{n}) & & \end{array}$


Table 12. Bond Lengths $(\AA)$

\begin{tabular}{|c|c|c|c|c|c|}
\hline atom & atom & distance & atom & atom & distánce \\
\hline$C(2)$ & $\mathrm{H}(1)$ & 0.95 & $C(2)$ & $\mathrm{H}(2)$ & 0.96 \\
\hline$C(2)$ & $\mathrm{H}(3)$ & 0.94 & $C(3)$ & $\mathrm{H}(4)$ & 0.95 \\
\hline$C(4)$ & $\mathrm{H}(5)$ & 0.99 & $c(5)$ & $\mathrm{H}(6)$ & $0.97^{\circ}$ \\
\hline$c(6)$ & $\mathrm{H}(7)$ & 0.96 & $c(7)$ & $\mathrm{H}(8)$ & 0.94 \\
\hline$C(9)$ & $\mathrm{H}(9)$ & 0.96 & $C(10)$ & $\mathrm{H}(10)$ & 0.95 \\
\hline$C\left(\begin{array}{ll}1 & 1\end{array}\right)$ & $\mathrm{H}(11)$ & 0.95 & $C(12)$ & $\mathrm{H}(12)$ & 0.95 \\
\hline$c(13)$ & $\mathrm{H}(13)$ & 0.96 & $c(15)$ & $\mathrm{H}(14)$ & 0.95 \\
\hline$c(16)$ & $\mathrm{H}(15)$ & 0.95 & $c(17)$ & $\mathrm{H}(16)$ & 0.96 \\
\hline$C(18)$ & $\mathrm{H}(17)$ & 0.94 & $C(19)$ & $\mathrm{H}(18)$ & 0.95 \\
\hline$c(21)$ & $\mathrm{H}(19)$ & 0.95 & $c(22)$ & $\mathrm{H}(20)$ & 0.95 \\
\hline$c(23)$ & $\mathrm{H}(21)$ & 0.94 & $c(24)$ & $\mathrm{H}(22)$ & 0.96 \\
\hline$C(25)$ & $\mathrm{H}(23)$ & 0.96 & $C(27)$ & $\mathrm{H}(24)$ & 0.94 \\
\hline$c(28)$ & $\mathrm{H}(25)$ & 0.95 & $c(29)$ & $\mathrm{E}(26)$ & 0.96 \\
\hline$c(30)$ & H ( 27) & 0.94 & $\mathrm{C}(31)$ & $\mathrm{H}(28)$ & 0.95 \\
\hline$c(33)$ & $\mathrm{H}(29)$ & 0.95 & $C(34)$ & $\mathrm{H}(30)$ & 0.96 \\
\hline$c(35)$ & $\mathrm{H}(31)$ & 0.96 & $c(36)$ & $\mathrm{H}(32)$ & 0.97 \\
\hline$c(37)$ & $\mathrm{H}(33)$ & 0.96 & $c(39)$ & $\mathrm{H}(34)$ & 0.97 \\
\hline$C(40)$ & $\mathrm{H}(35)$ & 0.97 & $C(41)$ & $\mathrm{H}(36)$ & 0.95 \\
\hline$c(42)$ & $\mathrm{H}(37)$ & 0.94 & $C(43)$ & $\mathrm{H}(38)$ & 0.95 \\
\hline
\end{tabular}


Table 13. Bond Angles $\left({ }^{\circ}\right)$

\begin{tabular}{|c|c|c|c|c|c|c|c|}
\hline atom & atom & atom & angle & atom & atom & atom & angle \\
\hline$P(1)$ & $\mathrm{Fe}(1)$ & $c(1)$ & $94.5(2)$ & $\mathrm{P}(1)$ & $\operatorname{Fe}(1)$ & $c(2)$ & $92.8(2)$ \\
\hline$P(1)$ & $\mathrm{Fe}(1)$ & $c(3)$ & $138.2(2)$ & $P(1)$ & $\operatorname{Fe}(1)$ & $C(4)$ & $161.1(2)$ \\
\hline$P(1)$ & $\mathrm{Fe}(1)$ & $c(5)$ & $122.0(2)$ & $P(1)$ & $\mathrm{Fe}(1)$ & $c(6)$ & $94.4(2)$ \\
\hline$P(1)$ & $\mathrm{Fe}(1)$ & $c(7)$ & $102.7(2)$ & $C(1)$ & $\mathrm{Fe}(1)$ & $c(2)$ & $90.9(3)$ \\
\hline$C(1)$ & $\mathrm{Fe}(1)$ & $c(3)$ & $127.2(3)$ & $c(1)$ & $\mathrm{Fe}(1)$ & $C(4)$ & $93.8(3)$ \\
\hline$C(1)$ & $\mathrm{Fe}(1)$ & $C(5)$ & $92.2(3)$ & $C(1)$ & $\mathrm{Fe}(1)$ & $c(6)$ & $124.3(3)$ \\
\hline$c(1)$ & $\mathrm{Fe}(1)$ & $c(7)$ & $156.9(3)$ & $c(2)$ & Fe(1) & $c(3)$ & $84.6(3)$ \\
\hline$C(2)$ & $\mathrm{Fe}(1)$ & $C(4)$ & $103.9(3)$ & $C(2)$ & $\mathrm{Fe}(1)$ & $C(5)$ & $144.6(3)$ \\
\hline$c(2)$ & $\mathrm{Fe}(1)$ & $c(6)$ & $143.2(3)$ & $c(2)$ & $\mathrm{Fe}(1)$ & $C(7)$ & $103.3(3)$ \\
\hline$c(3)$ & $\mathrm{Fe}(1)$ & $C(4)$ & $38 . .2(3)$ & $c(3)$ & $\mathrm{Fe}(1)$ & $c(5)$ & $65.7(3)$ \\
\hline$c(3)$ & $\mathrm{Fe}(1)$ & $c(6)$ & $66.3(3)$ & $c(3)$ & $\mathrm{Fe}(1)$ & $c(7)$ & $38.8(3)$ \\
\hline$C(4)$ & $\mathrm{Fe}(1)$ & $c(5)$ & $40.7(3)$ & $C(4)$ & $\mathrm{Fe}(1)$ & $c(6)$ & $67.0(3)$ \\
\hline$C(4)$ & $\mathrm{Fe}(1)$ & $c(7)$ & $65.4(3)$ & $c(5)$ & $\mathrm{Fe}(1)$ & $c(6)$ & $39.1(3)$ \\
\hline$C(5)$ & $\mathrm{Fe}(1)$ & $c(7)$ & $65.7(3)$ & $c(6)$ & $\mathrm{Fe}(1)$ & $c(7)$ & $40.0(3)$ \\
\hline $\operatorname{Fe}(1)$ & $P(1)$ & $c(8)$ & $113.1(2)$ & $\mathrm{Fe}(1)$ & $P(1)$ & $c(14)$ & $116.1(2)$ \\
\hline $\mathrm{Fe}(1)$ & $P(1)$ & $c(20)$ & $119.3(2)$ & $C(8)$ & $P(1)$ & $C(14)$ & $104.1(3)$ \\
\hline$c(8)$ & $P(1)$ & $c(20)$ & $103.0(2)$ & $C(14)$ & $P(1)$ & $c(20)$ & $99.1(2)$ \\
\hline $\mathrm{N}(1)$ & Si(1) & $c(26)$ & $108.1(3)$ & $\mathrm{N}(1)$ & si(1) & $c(32)$ & $109.4(3)$ \\
\hline$N(1)$ & $\operatorname{Si}(1)$ & $c(38)$ & $107.4(3)$ & $C(26)$ & $\mathrm{Si}(1)$ & $c(32)$ & $108.6(3)$ \\
\hline$c(26)$ & Si(1) & $c(38)$ & $112.9(2)$ & $c(32)$ & $\mathrm{Si}(1)$ & $c(38)$ & $110.4(2)$ \\
\hline Si(1) & $N(1)$ & $c(1)$ & $156.7(5)$ & $\mathrm{Fe}(1)$ & $\mathrm{C}(1)$ & $N(1)$ & $173.5(5)$ \\
\hline Fe(1) & $c(3)$ & $c(4)$ & $69.9(4)$ & $\mathrm{Fe}(1)$ & $c(3)$ & $c(7)$ & $71.3(4)$ \\
\hline$c(4)$ & $c(3)$ & $c(7)$ & $109.8(6)$ & $\mathrm{Fe}(1)$ & $C(4)$ & $c(3)$ & $71.9(4)$ \\
\hline$F e(1)$ & $c(4)$ & $c(5)$ & $69.8(4)$ & $c(3)$ & $C(4)$ & $C(5)$ & $107.6(7)$ \\
\hline $\mathrm{Fe}(1)$ & $C(5)$ & $c(4)$ & $69.5(3)$ & $\mathrm{Fe}(1)$ & $c(5)$ & $c(6)$ & $70.7(3)$ \\
\hline$C(4)$ & $C(5)$ & $c(6)$ & $108.1(6)$ & $\mathrm{Fe}(1)$ & $c(6)$ & $C(5)$ & $70.2(4)$ \\
\hline $\mathrm{Fe}(1)$ & $c(6)$ & $C(7)$ & $71.3(3)$ & $C(5)$ & $c(6)$ & $c(7)$ & $107.2(6)$ \\
\hline Fe(1) & $c(7)$ & $c(3)$ & $69.8(4)$ & $\mathrm{Fe}(1)$ & $C(7)$ & $c(6)$ & $68.7(3)$ \\
\hline$c(3)$ & $c(7)$ & $c(6)$ & $107.3(6)$ & $P(1)$ & $C(8)$ & $C(9)$ & $117.2(4)$ \\
\hline$P(1)$ & $C(8)$ & $C(13)$ & $124.1(4)$ & $C(9)$ & $c(8)$ & $C(13)$ & $118.3(5)$ \\
\hline$C(8)$ & $C(9)$ & $c(10)$ & $121.0(6)$ & $C(9)$ & $c(10)$ & $C(11)$ & $120.6(6)$ \\
\hline$c(10)$ & $C(11)$ & $c(12)$ & $118.0(6)$ & $c(11)$ & $C(12)$ & $C(13)$ & $122.0(6)$ \\
\hline$C(8)$ & $C(13)$ & $C(12)$ & $120.1(6)$ & $P(1)$ & $C(14)$ & $C(15)$ & $118.2(4)$ \\
\hline$P(1)$ & $C(14)$ & $C(19)$ & $123.5(4)$ & $c(15)$ & $C(14)$ & $C(19)$ & $118.1(5)$ \\
\hline$C(14)$ & $C(15)$ & $C(16)$ & $120.5(6)$ & $C(15)$ & $C(16)$ & $c(17)$ & $120.3(6)$ \\
\hline$c(16)$ & $c(17)$ & $C(18)$ & $120.6(6)$ & $c(17)$ & $C(18)$ & $c(19)$ & $119.2(6)$ \\
\hline
\end{tabular}


(C) 2003 American Chemical Society, Organometallics, Nakazawa om0208319 Supporting Info Page 25

Table 13. Bond Angles( ${ }^{\circ}$ ) (continued)

$\begin{array}{llllllll}\text { atom } & \text { atom } & \text { atom } & \text { angle } & \text { atom } & \text { atom } & \text { atom } & \text { angle } \\ \mathrm{C}(14) & \mathrm{C}(19) & \mathrm{C}(18) & 121.2(6) & \mathrm{P}(1) & \mathrm{C}(20) & \mathrm{C}(21) & 124.1(5) \\ \mathrm{P}(1) & \mathrm{C}(20) & \mathrm{C}(25) & 117.3(4) & \mathrm{C}(21) & \mathrm{C}(20) & \mathrm{C}(25) & 118.6(5) \\ \mathrm{C}(20) & \mathrm{C}(21) & \mathrm{C}(22) & 120.5(6) & \mathrm{C}(21) & \mathrm{C}(22) & \mathrm{C}(23) & 120.1(6) \\ \mathrm{C}(22) & \mathrm{C}(23) & \mathrm{C}(24) & 119.5(6) & \mathrm{C}(23) & \mathrm{C}(24) & \mathrm{C}(25) & 121.7(6) \\ \mathrm{C}(20) & \mathrm{C}(25) & \mathrm{C}(24) & 119.6(6) & \mathrm{S} i(1) & \mathrm{C}(26) & \mathrm{C}(27) & 121.3(5) \\ \mathrm{Si}(1) & \mathrm{C}(26) & \mathrm{C}(31) & 121.3(5) & \mathrm{C}(27) & \mathrm{C}(26) & \mathrm{C}(31) & 117.4(6) \\ \mathrm{C}(26) & \mathrm{C}(27) & \mathrm{C}(28) & 121.0(7) & \mathrm{C}(27) & \mathrm{C}(28) & \mathrm{C}(29) & 119.0(6) \\ \mathrm{C}(28) & \mathrm{C}(29) & \mathrm{C}(30) & 122.2(6) & \mathrm{C}(29) & \mathrm{C}(30) & \mathrm{C}(31) & 117.8(7) \\ \mathrm{C}(26) & \mathrm{C}(31) & \mathrm{C}(30) & 122.5(7) & \mathrm{S}(1) & \mathrm{C}(32) & \mathrm{C}(33) & 120.5(5) \\ \mathrm{Si}(1) & \mathrm{C}(32) & \mathrm{C}(37) & 122.0(4) & \mathrm{C}(33) & \mathrm{C}(32) & \mathrm{C}(37) & 117.4(6) \\ \mathrm{C}(32) & \mathrm{C}(33) & \mathrm{C}(34) & 121.5(6) & \mathrm{C}(33) & \mathrm{C}(34) & \mathrm{C}(35) & 120.1(5) \\ \mathrm{C}(34) & \mathrm{C}(35) & \mathrm{C}(36) & 119.9(6) & \mathrm{C}(35) & \mathrm{C}(36) & \mathrm{C}(37) & 120.0(6) \\ \mathrm{C}(32) & \mathrm{C}(37) & \mathrm{C}(36) & 121.0(6) & \mathrm{S} i(1) & \mathrm{C}(38) & \mathrm{C}(39) & 123.1(4) \\ \mathrm{Si}(1) & \mathrm{C}(38) & \mathrm{C}(43) & 119.4(5) & \mathrm{C}(39) & \mathrm{C}(38) & \mathrm{C}(43) & 117.5(6) \\ \mathrm{C}(38) & \mathrm{C}(39) & \mathrm{C}(40) & 123.0(6) & \mathrm{C}(39) & \mathrm{C}(40) & \mathrm{C}(41) & 117.8(7) \\ \mathrm{C}(40) & \mathrm{C}(41) & \mathrm{C}(42) & 120.9(6) & \mathrm{C}(41) & \mathrm{C}(42) & \mathrm{C}(43) & 121.5(7) \\ \mathrm{C}(38) & \mathrm{C}(43) & \mathrm{C}(42) & 119.3(7) & & & & \end{array}$


Table 14. Bond Angles $\left({ }^{\circ}\right)$

\begin{tabular}{|c|c|c|c|c|c|c|c|}
\hline atom & atom & atom & angle & atom & atom & atom & angle \\
\hline $\mathrm{Fe}(1)$ & $c(2)$ & $\mathrm{H}(1)$ & 109.8 & $\mathrm{Fe}(1)$ & $c(2)$ & $\mathrm{H}(2)$ & 109.4 \\
\hline $\mathrm{Fe}(1)$ & $c(2)$ & $\mathrm{H}(3)$ & 109.9 & $\mathrm{H}(1)$ & $c(2)$ & $\mathrm{H}(2)$ & 108.7 \\
\hline $\mathrm{H}(1)$ & $c(2)$ & $\mathrm{H}(3)$ & 110.1 & $\mathrm{H}(2)$ & $c(2)$ & $\mathrm{H}(3)$ & 109.0 \\
\hline $\mathrm{Fe}(1)$ & $c(3)$ & $\mathrm{H}(4)$ & 125.1 & $c(4)$ & $c(3)$ & $\mathrm{H}(4)$ & 126.1 \\
\hline$c(7)$ & $c(3)$ & $\mathrm{H}(4)$ & 124.1 & $\mathrm{Fe}(1)$ & $c(4)$ & $\mathrm{H}(5)$ & 126.7 \\
\hline$c(3)$ & $C(4)$ & $\mathrm{H}(5)$ & 126.6 & $C(5)$ & $C(4)$ & $\mathrm{H}(5)$ & 125.7 \\
\hline $\mathrm{Fe}(1)$ & $c(5)$ & $H(6)$ & 126.0 & $C(4)$ & $C(5)$ & $\mathrm{H}(6)$ & 127.3 \\
\hline$c(6)$ & $c(5)$ & $\mathrm{H}(6)$ & 124.7 & $\mathrm{Fe}(1)$ & $C(6)$ & $\mathrm{H}(7)$ & 127.1 \\
\hline$c(5)$ & $C(6)$ & $\mathrm{H}(7)$ & 127.1 & $c(7)$ & $c(6)$ & $\mathrm{H}(7)$ & 125.5 \\
\hline $\mathrm{Fe}(1)$ & $c(7)$ & $\mathrm{H}(8)$ & 126.6 & $c(3)$ & $c(7)$ & $\mathrm{H}(8)$ & 128.0 \\
\hline$c(6)$ & $c(7)$ & $\mathrm{H}(8)$ & 124.7 & $c(8)$ & $c(9)$ & $\mathrm{H}(9)$ & 118.8 \\
\hline$c(10)$ & $c(9)$ & $\mathrm{H}(9)$ & 120.2 & $C(9)$ & $c(10)$ & $\mathrm{H}(10)$ & 119.1 \\
\hline$C(11)$ & $c(10)$ & $\mathrm{H}(10)$ & 120.3 & $c(10)$ & $\mathrm{C}(11)$ & $\mathrm{H}(11)$ & 122.2 \\
\hline$C(12)$ & $C(11)$ & $\mathrm{H}(11)$ & 119.8 & $C(11)$ & $c(12)$ & $\mathrm{H}(12)$ & 119.9 \\
\hline$C(13)$ & $C(12)$ & $\mathrm{H}(12)$ & 118.1 & $C(8)$ & $c(13)$ & $\mathrm{H}(13)$ & 120.0 \\
\hline$C(12)$ & $c(13)$ & $\mathrm{H}(13)$ & 119.9 & $C(14)$ & $C(15)$ & $\mathrm{H}(14)$ & 120.9 \\
\hline$C(16)$ & $c(15)$ & $\mathrm{H}(14)$ & 118.5 & $c(15)$ & $c(16)$ & $\mathrm{H}(15)$ & 119.7 \\
\hline$C(17)$ & $C(16)$ & $\mathrm{H}(15)$ & 120.0 & $c(16)$ & $c(17)$ & $\mathrm{H}(16)$ & 118.0 \\
\hline$c(18)$ & $C(17)$ & $\mathrm{H}(16)$ & 121.4 & $c(17)$ & $C(18)$ & $\mathrm{H}(17)$ & 118.8 \\
\hline$c(19)$ & $C(18)$ & H (17) & 122.0 & $c(14)$ & $c(19)$ & $\mathrm{H}(18)$ & 119.6 \\
\hline$C(18)$ & $C(19)$ & $\mathrm{H}(18)$ & 119.2 & $c(20)$ & $c(21)$ & $\mathrm{H}(19)$ & 119.6 \\
\hline$C(22)$ & $C(21)$ & $\mathrm{H}(19)$ & 119.9 & $c(21)$ & $C(22)$ & $\mathrm{H}(20)$ & 120.5 \\
\hline$c(23)$ & $c(22)$ & $\mathrm{H}(20)$ & 119.4 & $c(22)$ & $c(23)$ & $\mathrm{H}(21)$ & 120.7 \\
\hline$C(24)$ & $c(23)$ & $\mathrm{H}(21)$ & 119.7 & $C(23)$ & $c(24)$ & $\mathrm{H}(22)$ & 118.8 \\
\hline$C(25)$ & $C(24)$ & $\mathrm{H}(22)$ & 119.5 & $C(20)$. & $C(25)$ & $\mathrm{H}(23)$ & 120.7 \\
\hline$C(24)$ & $C(25)$ & $\mathrm{H}(23)$ & 119.7 & $c(26)$ & $c(27)$ & $\mathrm{E}(24)$ & 119.6 \\
\hline$c(28)$ & $c(27)$ & $\mathrm{H}(24)$ & 119.4 & $c(27)$ & $c(28)$ & $\mathrm{H}(25)$ & 120.5 \\
\hline$C(29)$ & $c(28)$ & $\mathrm{H}(25)$ & 120.6 & $c(28)$ & $C(29)$ & $\mathrm{H}(26)$. & 119.3 \\
\hline$C(30)$ & $C(29)$ & $\mathrm{H}(26)$ & 118.5 & $C(29)$ & $c(30)$ & $\mathrm{H}(27)$ & 121.9 \\
\hline$c(31)$ & $c(30)$ & $\mathrm{H}(27)$ & 120.2 & $C(26)$ & $C(31)$ & $\mathrm{H}(28)$ & 119.9 \\
\hline$c(30)$ & $c(31)$ & $\mathrm{H}(28)$ & 117.5 & $c(32)$ & $c(33)$ & $\mathrm{H}(29)$. & 119.6 \\
\hline$C(34)$ & $c(33)$ & $\mathrm{H}(29)$ & 118.9 & $c(33)$ & $C(34)$ & $\mathrm{H}(30)$ & 120.4 \\
\hline$c(35)$ & $C(34)$ & $\mathrm{H}(30)$ & 119.5 & $c(34)$ & $c(35)$ & $\mathrm{H}(31)$ & 120.2 \\
\hline$c(36)$ & $c(35)$ & $\mathrm{H}(31)$ & 119.9 & $c(35)$ & $c(36)$ & $\mathrm{H}(32)$ & 120.9 \\
\hline$c(37)$ & $c(36)$ & $\mathrm{H}(32)$ & 119.1 & $c(32)$ & $c(37)$ & $\mathrm{H}(33)$ & 120.1 \\
\hline$C(36)$ & $C(37)$ & $\mathrm{H}(33)$ & 118.9 & $c(38)$ & $C(39)$ & $\mathrm{H}(34)$ & 117.9 \\
\hline
\end{tabular}




\begin{tabular}{|c|c|c|c|c|c|c|c|}
\hline atom & a tom & atom & angle & atom & atom & a tom & angle \\
\hline$C(40)$ & $c(39)$ & $\mathrm{H}(34)$ & 119.1 & $c(39)$ & $C(40)$ & $\mathrm{H}(35)$ & 120.4 \\
\hline$C(41)$ & $C(40)$ & $\mathrm{H}(35)$ & 121.8 & $C(40)$ & $c(41)$ & $\mathrm{H}(36)$ & 118.7 \\
\hline$C(42)$ & $C(41)$ & $\mathrm{H}(36)$ & 120.4 & $C(41)$ & $c(42)$ & $\mathrm{H}(37)$ & 119.5 \\
\hline$C(43)$ & $c(42)$ & $\mathrm{H}(37)$ & 119.0 & $C(38)$ & $c(43)$ & $\mathrm{H}(38)$ & 119.3 \\
\hline$c(42)$ & $c(43)$ & $\mathrm{H}(38)$ & 121.4 & & & & \\
\hline
\end{tabular}


Table 15. Torsion Angles $\left(^{\circ}\right)$

\begin{tabular}{|c|c|c|c|c|c|c|c|c|c|}
\hline & & & & & & & & & \\
\hline 1) & $P(1)$ & $c(8)$ & $C(9)$ & $-56.5(5)$ & (1) & 1) & 81 & (3) & \\
\hline ra & $P(1)$ & $C(14)$ & $C(15)$ & -12. & & & & & \\
\hline (1) & $P(1)$ & $c(20)$ & $c(21)$ & & & & & s & \\
\hline (1) & $c(1)$ & $\mathrm{N}(1)$ & $i(1)$ & & & & & , & \\
\hline$(1)$ & $c(3)$ & & & & & & & & \\
\hline & $C(4)$ & & & & & & & & \\
\hline & $c(5)$ & & & & & & & & \\
\hline & $c(6)$ & & & & & $c(7)$ & & & \\
\hline & $c(7)$ & $c(6)$ & Cl & -61 & & & & & \\
\hline & $\mathrm{Fel}$ & Cl & & & & & & & \\
\hline & $\mathrm{Fel}$ & cl & & & & & & & \\
\hline & ) & & & & & & & & \\
\hline & & & & & & & & & \\
\hline & & & & & & & & & \\
\hline & & C & & & & & & & 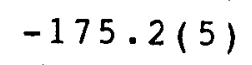 \\
\hline & & & & & & & & & -1 \\
\hline & & & & & & & & & \\
\hline & & $c$ & & & & & & & \\
\hline & & & & & & & & & \\
\hline & & & & & & & & & -1 \\
\hline & & & & & & & & & \\
\hline & & & & & & & & & \\
\hline & & & & & & & & & \\
\hline & & & & & & & & & \\
\hline & & & & & & & & & \\
\hline & & & & & & & & & \\
\hline & & & & & & & & & \\
\hline & & & & & & & & & \\
\hline (1) & & & & & & & & $c(3)$ & \\
\hline (1) & 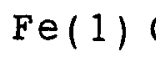 & & & & & & & & \\
\hline (1) & ( & & & & & & & & \\
\hline 1 & & & & & & & & & \\
\hline & & & & & & & & & \\
\hline & & & & 20 & & & & & \\
\hline & & & & 96. & & & & & 143.313 \\
\hline & & & $C(20)$ & $-24.8(3)$ & -10, & & & $-(4)$ & 120.713 \\
\hline
\end{tabular}


Table 15. Torsion Angles $\left({ }^{\circ}\right.$ ) (continued)

\begin{tabular}{|c|c|c|c|c|c|c|c|c|c|}
\hline tom & tom & $\mathrm{m}$ & tom & angle & & & atom & & \\
\hline$(2)$ & $e(1)$ & $c(3)$ & (7) & $-118.9(4)$ & (2) & & $C(4)$ & $c(3)$ & $-61.9(5)$ \\
\hline (2). & $(1)$ & $C(4)$ & (5) & -179. & & & $C(5)$ & & $1.5(7)$ \\
\hline 8 & $e(1)$ & $C(5)$ & $(6)$ & -117 & & & $c(6)$ & & $0.9(5)$ \\
\hline (2) & $e(1)$ & $c(6)$ & $(7)$ & $3.9(7)$ & 2) & & & & (4) \\
\hline (2) & & $c(7)$ & $(6)$ & $-177.6(4)$ & & & & 8) & 3) \\
\hline & $(1)$ & $P(1)$ & $C(14)$ & $131.6(3)$ & (3) & & 1) & $(20)$ & 3) \\
\hline & 1) & $C(4)$ & $C(5)$ & $-117.3(7)$ & (3) & & $c(5)$ & (4) & 4) \\
\hline ) & & (5) & $(6)$ & -81 & $3)$ & & $c(6)$ & (5) & 4) \\
\hline & & ) & & & & 1) & $c(7)$ & 6) & 118 \\
\hline & 1 & $e(1)$ & 5) & 117. & & & $\mathrm{Fe}(1)$ & 6) & \\
\hline & 4) & e (1) & $7)$ & 36. & & & & & 7) \\
\hline & 7) & $e(1)$ & 4) & -35 & & & (1) & $c(5)$ & -80 \\
\hline & (7) & $\mathrm{Fe}(1)$ & $c(6)$ & $-118.9(6)$ & & & 6) & 5) & -2 \\
\hline & $e(1)$ & $P(1)$ & $C(8)$ & $-56.5(7)$ & & & 1) & & 63 \\
\hline & ) & ) & 1 & -177 & & & 3) & & 120 \\
\hline & & $c(5)$ & $c(6)$ & -1.18 & & 1) & 6) & & \\
\hline & ) & 6) & & -78 & & 1) & $c(7)$. & $c(6)$ & 83 \\
\hline & & & & & & & $e(1)$ & $c(6)$ & -82 \\
\hline b) & & 1 & 7) & -120 & & & 7) & 6) & \\
\hline 4) & ) & $\mathrm{Fe}(1)$ & $c(6)$ & 118 & & & & & \\
\hline 4) & j) & $c(6)$ & $c(7)$ & 2. & & & 1 & & -76 \\
\hline ) & & $P(1)$ & $C(14)$ & 43 & & & 1) & $c(20)$ & 161 \\
\hline ) & & & & 80. & & 1) & $c(6)$ & $c(7)$ & -116. \\
\hline (5) & 1 & $c(7)$ & & & & & (1) & $c(6)$ & -36 \\
\hline ( 5 ) & $C(4)$ & $\mathrm{Fe}(1)$ & $c(7)$ & -80.7 & & & & & \\
\hline (5) & $c(6)$ & $\mathrm{Fe}(1)$ & $c(7)$ & 116.9 & & & & & -47 \\
\hline (6) & & $P(1)$ & $c(14)$ & $72.8(3)$ & 5) & 1) & $P(1)$ & & -168 \\
\hline (6) & & $c(3)$ & $c(7)$ & $37.9(4)$ & & $C(5)$ & $\mathrm{Fe}(1)$ & $\mathrm{C}($ & -38 \\
\hline & & $P($ & $C(8)$ & $-7.8(3)$ & & (1) & $P(1)$ & $C(14)$ & 112. \\
\hline (7 & $\mathrm{Fe}$ & $P(1)$ & $c(20)$ & $-129.1(3)$ & & & $c(14)$ & $c(15)$ & 112.5 \\
\hline (8) & $P(1)$ & $C(14)$ & $C(19)$ & $-71 \cdot 3$ & & & $c(20)$ & $C(21)$ & (5) \\
\hline$(8)$ & $P(1)$ & $C(20)$ & $C(25)$ & $178.9(5)$ & & & $(10)$ & 1) & $-1(1)$ \\
\hline (8) & $c(13)$ & $C(12)$ & $\mathrm{C}(11)$ & $2(1)$ & & & $P(1)$ & $c(14)$ & $176.7(4)$ \\
\hline (9) & $C(8)$ & $P(1)$ & $c(20)$ & $73.7(5$ & $C(9)$ & & $c(13)$ & $C(12)$ & $-2.5(10)$ \\
\hline (9) & $c(10)$ & $C(11)$ & $c(12)$ & $1(1)$ & $C(10)$ & & $C(8)$ & $c(13)$ & $1.6(9)$ \\
\hline$(10)$ & $C(11)$ & $C(12)$ & $c(13)$ & $-2(1)$ & $C(13)$ & $c(8)$ & $P(1)$ & $C(14)$ & $-10.5(6)$ \\
\hline
\end{tabular}




\begin{tabular}{|c|c|c|c|c|c|c|c|c|c|}
\hline atom & atom & atom & atom & angle & a tom & atom & atom & atom & angle \\
\hline$c(13)$ & $c(8)$ & $P(1)$ & $c(20)$ & $-113 \cdot 5(5)$ & $c(14)$ & $P(1)$ & $c(20)$ & $c(21)$ & $-105 \cdot 1(5)$ \\
\hline$c(14)$ & $P(1)$ & $c(20)$ & $c(25)$ & $72.1(5)$ & $c(14)$ & $c(15)$ & $c(16)$ & $c(17)$ & $-1(1)$ \\
\hline$c(14)$ & $c(19)$ & $C(18)$ & $c(17)$ & $1.1(10)$ & $C(15)$ & $\mathrm{C}(14)$ & $P(1)$ & $c(20)$ & $-141.1(5)$ \\
\hline$c(15)$ & $C(14)$ & $c(19)$ & $C(18)$ & $-1.6(9)$ & $c(15)$ & $C(16)$ & $c(17)$ & $c(18)$ & $1(1)$ \\
\hline$c(16)$ & $c(15)$ & $C(14)$ & $C(19)$ & $1.8(9)$ & $c(16)$ & $C(17)$ & $c(18)$ & $c(19)$ & $0(1)$ \\
\hline$c(19)$ & $c(14)$ & $P(1)$ & $c(20)$ & $34.6(5)$ & $c(20)$ & $C(21)$ & $C(22)$ & $c(23)$ & $-1(1)$ \\
\hline$c(20)$ & $c(25)$ & $c(24)$ & $C(23)$ & $0(1)$ & $C(21)$ & $c(20)$ & $c(25)$ & $C(24)$ & $-1.2(10)$ \\
\hline$c(21)$ & $c(22)$ & $c(23)$ & $C(24)$ & $0(1)$ & $C(22)$ & $c(21)$ & $C(20)$ & $C(25)$ & $1.7(9)$ \\
\hline$c(22)$ & $c(23)$ & $c(24)$ & $C(25)$ & $0(1)$ & $c(26)$ & $\mathrm{Si}(1)$ & $c(32)$ & $c(33)$ & $66.5(5)$ \\
\hline$c(26)$ & Si(1) & $c(32)$ & $c(37)$ & $-114.2(5)$ & $c(26)$ & Si(1) & $C(38)$ & $c(39)$ & $30.5(6)$ \\
\hline$c(26)$ & Si(1) & $C(38)$ & $c(43)$ & $-148 \cdot 3(5)$ & $c(26)$ & $c(27)$ & $c(28)$ & $C(29)$ & $1.4(10)$ \\
\hline$c(26)$ & $c(31)$ & $c(30)$ & $C(29)$ & $1(1)$ & $c(27)$ & $c(26)$ & si(1) & $c(32)$ & $-146.1(5)$ \\
\hline$c(27)$ & $c(26)$ & Si(1) & $c(38)$ & $91.1(5)$ & $c(27)$ & $C(26)$ & $c(31)$ & $c(30)$ & $-1.5(9)$ \\
\hline$c(27)$ & $c(28)$ & $C(29)$ & $c(30)$ & $-1(1)$ & $C(28)$ & $c(27)$ & $c(26)$ & $c(31)$ & $-0.2(9)$ \\
\hline$C(28)$ & $C(29)$ & $c(30)$ & $c(31)$ & $0(1)$ & $c(31)$ & $c(26)$ & Si(1) & $c(32)$ & $34.6(5)$ \\
\hline$C(31)$ & $c(26)$ & si(1) & $c(38)$ & $-88.2(5)$ & $c(32)$ & Si(1) & $c(38)$ & $c(39)$ & $-91 \cdot 3(6)$ \\
\hline$c(32)$ & Si(1) & $c(38)$ & $C(43)$ & $89.9(6)$ & $c(32)$ & $c(33)$ & $C(34)$ & $c(35)$ & $-0.4(10)$ \\
\hline$c(32)$ & $c(37)$ & $c(36)$ & $c(35)$ & $0(1)$ & $c(33)$ & $c(32)$ & Si(1) & $c(38)$ & $-169.2(5)$ \\
\hline$c(33)$ & $c(32)$ & $c(37)$ & $c(36)$ & $0(1)$ & $c(33)$ & $C(34)$ & $c(35)$ & $c(36)$ & $0(1)$ \\
\hline$c(34)$ & $c(33)$ & $c(32)$ & $c(37)$ & $0.7(9)$ & $c(34)$ & $c(35)$ & $c(36)$ & $c(37)$ & $0(1)$ \\
\hline$c(37)$ & $c(32)$ & Si(1) & $c(38)$ & $10.1(6)$ & $c(38)$ & $C(39)$ & $c(40)$ & $C(41)$ & $1(1)$ \\
\hline$c(38)$ & $C(43)$ & $c(42)$ & $c(41)$ & $-2(1)$ & $c(39)$ & $c(38)$ & $c(43)$ & $C(42)$ & $1(1)$ \\
\hline$c(39)$ & $C(40)$ & $c(41)$ & $c(42)$ & $-1(1)$ & $c(40)$ & $c(39)$ & $c(38)$ & $c(43)$ & $0(1)$ \\
\hline$c(40)$ & $c(41)$ & $C(42)$ & $c(43)$ & $2(1)$ & & & & & \\
\hline
\end{tabular}


(C) 2003 American Chemical Society, Organometallics, Nakazawa om0208319 Supporting Info Page 31

\begin{tabular}{|c|c|c|c|c|c|}
\hline atom & atom & distance & a tom & atom & distance \\
\hline$c(3)$. & $\left.c(38)^{1}\right)$ & $3.565(9)$ & $C(4)$ & $c(27)^{1)}$ & $3.247(9)$ \\
\hline$c(4)$ & $C(39)^{1)}$ & $3.57(1)$ & $c(5)$ & $\left.c(27)^{1}\right)$ & $3.475(9)$ \\
\hline$c(5)$ & $C(28)^{1)}$ & $3.551(9)$ & $c(7)$ & $\left.C(18)^{1}\right)$ & $3.572(10)$ \\
\hline$c(10)$ & $\left.c(34)^{2}\right)$ & $3.285(10)$ & $C(18)$ & $c(36)^{3)}$ & $3.574(10)$ \\
\hline
\end{tabular}

\section{Symmetry operations}
(1) $\quad X, Y-1, Z$
(3) $\quad X+1 / 2,-Y-1 / 2, Z+1 / 2$
(2) $\quad X,-Y-1, Z+1 / 2$ 
(C) 2003 American Chemical Society, Organometallics, Nakazawa om0208319 Supporting Info Page 32

\section{The theoretical results}

Table 17. Potential energies (E), ZPE-corrected potential energies (E+ZPE) and Gibbs free energies $(G)$ of the systems discussed in the manuscript in atomic unit (Level of theory: B3LYP; Basis set used: LanL2DZ for Fe and 6-31G(d) for $\mathrm{H}, \mathrm{C}, \mathrm{P}$, and $\mathrm{Si}$ ).

\begin{tabular}{cccc}
\hline System & E & E+ZPE & G \\
\hline $\mathbf{1 1}$ & -839.54848 & -839.34300 & -839.38453 \\
$\mathbf{1 2}$ & -972.33999 & -972.08689 & -972.13791 \\
$\mathbf{1 3}$ & -972.27928 & -972.02579 & -972.07238 \\
$\mathbf{1 4}$ & -972.32535 & -972.07155 & -972.11819 \\
$\mathbf{1 5}$ & -972.35394 & -972.09992 & -972.14873 \\
$\mathbf{1 6}$ & -972.36206 & -972.10928 & -972.15954 \\
$\mathbf{1 7}$ & -470.17724 & -470.04909 & -470.08415 \\
$\mathbf{1 8}$ & -972.33794 & -972.08575 & -972.13590 \\
$\mathbf{1 9}$ & -858.96587 & -858.72432 & -858.77218 \\
$\mathbf{2 0}$ & -858.94361 & -858.70233 & -858.74861 \\
$\mathbf{2 1}$ & -858.93391 & -858.69061 & -858.73346 \\
$\mathbf{2 2}$ & -858.95897 & -858.71653 & -858.76132 \\
$\mathbf{2 3}$ & -972.29537 & -972.04334 & -972.09514 \\
$\mathbf{2 4}$ & -972.30771 & -972.05677 & -972.10635 \\
$\mathbf{2 5}$ & -858.9469 & -858.70595 & -858.75209 \\
$\mathbf{2 6}$ & -858.9126 & -858.67320 & -858.71855 \\
$\mathbf{2 7}$ & -858.90726 & -858.66780 & -858.71420 \\
$\mathbf{2 8}$ & -858.96009 & -858.71786 & -858.76583 \\
TS1 & -972.25487 & -972.00237 & -972.04890 \\
TS2 & -972.31492 & -972.06267 & -972.11085 \\
TS3 & -972.31894 & -972.06503 & -972.11051 \\
TS4 & -972.32798 & -972.07586 & -972.12499 \\
TS5 & -470.17724 & -470.04909 & -470.08415 \\
TS6 & -858.93965 & -858.69982 & -858.74673 \\
TS8 & -858.91925 & -858.67847 & -858.72298 \\
TS9 & -972.28273 & -972.03318 & -972.08036 \\
TS10 & -858.90568 & -858.66620 & -858.71154 \\
TS11 & -858.90017 & -858.66043 & -858.70446 \\
TS12 & -858.90588 & -858.66691 & -858.71220 \\
\hline & & & \\
\hline
\end{tabular}


(C) 2003 American Chemical Society, Organometallics, Nakazawa om0208319 Supporting Info Page 33

\begin{tabular}{cccc}
\hline $\mathbf{M e}_{3} \mathrm{SiNC}$ & -502.11261 & -501.99272 & -502.02632 \\
$\mathbf{M e}_{3} \mathrm{SiCN}$ & -502.11949 & -501.99902 & -502.03245 \\
$\mathbf{C H}_{3} \mathbf{C N}$ & -132.75149 & -1.32 .70585 & -132.72882 \\
\hline
\end{tabular}

Cartesian coordinates of the optimized geometrics (Level: B3LYP; Basis sct used: LanL2DZ for $\mathrm{Fe}$ and 6-31G(d) for $\mathrm{H}, \mathrm{C}, \mathrm{P}$, and $\mathrm{Si}$ ) given in Chem3d 'cc2' format.

$\begin{array}{lrrrrrrrrr}11 & & & & & & & & & \\ \mathrm{Fe} & 1 & .5472520 & .2241870 & .2089580 & 266 & 2 & 4 & \\ \mathrm{C} & 2 & .1275200 & 1.9081020 & -.0105360 & 62 & 1 & 3 & & \\ \mathrm{O} & 3 & -.1011520 & 3.0313820 & -.1984230 & 81 & 2 & & & \\ \mathrm{Si} & 4 & -1.5852680 & -.3344500 & -.0578380 & 144 & 1 & 5 & 6 & 7 \\ \mathrm{C} & 5 & -2.1224620 & -2.0976120 & -.5292040 & 64 & 4 & 13 & 14 & 15 \\ \mathrm{C} & 6 & -3.0268220 & .8257270 & -.4830670 & 64 & 4 & 16 & 17 & 18 \\ \mathrm{C} & 7 & -1.1237140 & -.2264440 & 1.8210400 & 64 & 4 & 19 & 20 & 21 \\ \mathrm{C} & 8 & 1.4083910 & -1.1876520 & -1.1199350 & 63 & & 9 & 12 & 22 \\ \mathrm{C} & 9 & 1.5994150 & -1.6275580 & .2189960 & 63 & & 8 & 10 & 23 \\ \mathrm{C} & 10 & 2.3953610 & -.6476520 & .9025860 & 63 & & 9 & 11 & 24 \\ \mathrm{C} & 11 & 2.6807760 & .3965260 & -.0040700 & 63 & & 10 & 12 & 25 \\ \mathrm{C} & 12 & 2.0402390 & .0837560 & -1.2538070 & 63 & & 8 & 11 & 26 \\ \mathrm{H} & 13 & -3.1020980 & -2.3436420 & -.0986490 & 11 & 5 & & & \\ \mathrm{H} & 14 & -1.4018270 & -2.8471930 & -.1844760 & 11 & 5 & & & \\ \mathrm{H} & 15 & -2.2132210 & -2.1998510 & -1.6179220 & 11 & 5 & & & \\ \mathrm{H} & 16 & -3.9587400 & .4963590 & -.0051570 & 11 & 6 & & & \\ \mathrm{H} & 17 & -3.2040580 & .8405450 & -1.5658730 & 11 & 6 & & & \\ \mathrm{H} & 18 & -2.8262030 & 1.8557980 & -.1701350 & 11 & 6 & & & \\ \mathrm{H} & 19 & -1.1833060 & -1.2033990 & 2.3082050 & 11 & 7 & & & \\ \mathrm{H} & 20 & -1.7542560 & .5017500 & 2.3404640 & 11 & 7 & & & \\ \mathrm{H} & 21 & -.0798030 & .1460870 & 2.0387840 & 11 & 7 & & & \\ \mathrm{H} & 22 & .8626840 & -1.7108490 & -1.8927680 & 11 & 8 & & & \\ \mathrm{H} & 23 & 1.2234840 & -2.5478840 & .6465010 & 11 & 9 & & & \\ \mathrm{H} & 24 & 2.7021500 & -.6910450 & 1.9398770 & 11 & 10 & & & \\ \mathrm{H} & 25 & 3.2397860 & 1.2968920 & .2136660 & 11 & 11 & & & \\ \mathrm{H} & 26 & 2.0701300 & .6875480 & -2.1507460 & 11 & 12 & & & \\ & & & & & & & & \end{array}$


(C) 2003 American Chemical Society, Organometallics, Nakazawa om0208319 Supporting Info Page 34

12

$\begin{array}{lrrrrrrrrr}\mathrm{Fe} & 1 & .4783190 & -.5286180 & .2323560 & 266 & 2 & 4 & 8 & \\ \mathrm{C} & 2 & .3067650 & .0069990 & 1.8791160 & 62 & 1 & 3 & & \\ \mathrm{O} & 3 & .2392670 & .3659220 & 2.9829130 & 81 & 2 & & . & \\ \mathrm{Si} & 4 & -.3207940 & 1.6107060 & -.3125500 & 144 & 1 & 5 & 6 & 7 \\ \mathrm{C} & 5 & -.8015780 & 1.7180670 & -2.1650130 & 64 & 4 & 11 & 12 & 13 \\ \mathrm{C} & 6 & .9438970 & 3.0048120 & .0237370 & 64 & 4 & 14 & 15 & 16 \\ \mathrm{C} & 7 & -1.9021430 & 2.1176410 & .6403300 & 64 & 4 & 17 & 18 & 19 \\ \mathrm{~N} & 8 & -1.2832400 & -1.1622010 & .0583090 & 72 & 1 & 9 & & \\ \mathrm{C} & 9 & -2.3620330 & -1.5792660 & -.0496990 & 62 & 8 & 10 & & \\ \mathrm{C} & 10 & -3.7230410 & -2.0875620 & -.1804190 & 64 & 9 & 20 & 21 & 22 \\ \mathrm{H} & 11 & -1.2485600 & 2.6945550 & -2.3967710 & 11 & 5 & & & \\ \mathrm{H} & 12 & -1.5366460 & .9463880 & -2.4266410 & 11 & 5 . & & \\ \mathrm{H} & 13 & .0620320 & 1.5852010 & -2.8278680 & 11 & 5 & & & \\ \mathrm{H} & 14 & .5302600 & 3.9850330 & -.2493700 & 11 & 6 & & & \\ \mathrm{H} & 15 & 1.8732850 & 2.8702330 & -.5429150 & 11 & 6 & & & \\ \mathrm{H} & 16 & 1.2106260 & 3.0422680 & 1.0871720 & 11 & 6 & & & \\ \mathrm{H} & 17 & -2.2343260 & 3.1178670 & .3316770 & 11 & 7 & & & \\ \mathrm{H} & 18 & -1.7329890 & 2.1431380 & 1.7232490 & 11 & 7 & & & \\ \mathrm{H} & 19 & -2.7310680 & 1.4240910 & .4520350 & 11 & 7 & & & \\ \mathrm{H} & 20 & -4.3771630 & -1.5921080 & .5447690 & 11 & 10 & & & . \\ \mathrm{H} & 21 & -3.7522820 & -3.1666350 & .0054260 & 11 & 10 & & & \\ \mathrm{H} & 22 & -4.1105210 & -1.8945770 & -1.1866180 & 11 & 10 & & & \\ \mathrm{C} & 23 & 2.0534110 & -.2113420 & -1.1867960 & 63 & & 24 & 27 & 28 \\ \mathrm{C} & 24 & 1.3037770 & -1.3517690 & -1.5812700 & 63 & & 23 & 25 & 29 \\ \mathrm{C} & 25 & 1.4186340 & -2.3331040 & -.5416710 & 63 & .24 & 26 & 30 \\ \mathrm{C} & 26 & 2.2242750 & -1.7984400 & .4865720 & 63 & & 25 & 27 & 31 \\ \mathrm{C} & 27 & 2.6015630 & -.4677030 & .1040310 & 63 & . & 23 & 26 & 32 \\ \mathrm{H} & 28 & 2.1886340 & .6922420 & -1.7646210 & 11 & 23 & & & \\ \mathrm{H} & 29 & .7490870 & -1.4615640 & -2.5033520 & 11 & 24 & & & \\ \mathrm{H} & 30 & .9351180 & -3.3014050 & -.5285160 & 11 & 25 & & . & \\ \mathrm{H} & 31 & 2.4748440 & -2.2852840 & 1.4198430 & 11 & 26 & & & \\ \mathrm{H} & 32 & 3.2250610 & .2065950 & .6755400 & 11 & 27 & & & \\ & & & & & & & \end{array}$


(C) 2003 American Chemical Society, Organometallics, Nakazawa om0208319 Supporting Info Page 35

$\begin{array}{lrrrrrrrrr}\mathrm{Fe} & 1 & -.6587770 & .0447910 & -.0591970 & 266 & 2 & 4 & 9 & 15 \\ \mathrm{C} & 2 & -.0666430 & 1.2893530 & 1.0273270 & 62 & 1 & 3 & & \\ \mathrm{O} & 3 & .2169790 & 2.1182540 & 1.7781620 & 81 & 2 & & & \\ \mathrm{Si} & 4 & 1.8225670 & -.0568450 & -.1164560 & 1.44 & 1 & 5 & 6 & 7 \\ \mathrm{C} & 5 & 2.5267450 & -1.4451080 & -1.2231510 & 64 & 4 & 16 & 17 & 18 \\ \mathrm{C} & 6 & 2.4226720 & -.4616570 & 1.6522160 & 64 & 4 & 19 & 20 & 21 \\ \mathrm{C} & 7 & 2.6712470 & 1.5626480 & -.6172120 & 64 & 4 & 22 & 23 & 24 \\ \mathrm{~N} & 8 & -2.4759140 & 2.4037100 & -.8326260 & 71 & 9 & & & \\ \mathrm{C} & 9 & -1.7564990 & 1.5247010 & -.5591950 & 62 & 1 & 8 & & \\ \mathrm{C} & 10 & -.4053430 & -2.0254470 & .4608310 & 63 & & 11 & 14 & 25 \\ \mathrm{C} & 11 & -1.2115150 & -1.9467240 & -.7170330 & 63 & & 10 & 12 & 26 \\ \mathrm{C} & 12 & -2.3776940 & -1.2125600 & -.4050830 & 63 & & 11 & 13 & 27 \\ \mathrm{C} & 13 & -2.3120470 & -.8391520 & .9767680 & 63 & & 12 & 14 & 28 \\ \mathrm{C} & 14 & -1.1058510 & -1.3594410 & 1.5121840 & 63 & & 10 & 13 & 29 \\ \mathrm{C} & 15 & -.0305890 & .3996420 & -2.0152700 & 64 & 1 & 30 & 31 & 32 \\ \mathrm{H} & 16 & 3.4601370 & -1.8021880 & -.7689740 & 11 & 5 & & & \\ \mathrm{H} & 17 & 2.7707130 & -1.0720570 & -2.2231060 & 11 & 5 & & & \\ \mathrm{H} & 18 & 1.8660850 & -2.3091160 & -1.3448740 & 11 & 5 & & & \\ \mathrm{H} & 19 & 3.5194480 & -.5193680 & 1.6377790 & 11 & 6 & & & \\ \mathrm{H} & 20 & 2.0508770 & -1.4264410 & 2.0157900 & 11 & 6 & & & \\ \mathrm{H} & 21 & 2.1460290 & .3036850 & 2.3841950 & 11 & 6 & & & \\ \mathrm{H} & 22 & 3.7555280 & 1.4332970 & -.5000580 & 11 & 7 & & & \\ \mathrm{H} & 23 & 2.3690470 & 2.4058970 & .0120840 & 11 & 7 & & & \\ \mathrm{H} & 24 & 2.4798900 & 1.8310260 & -1.6600560 & 11 & 7 & & & \\ \mathrm{H} & 25 & .5168250 & -2.5778110 & .5637050 & 11 & 10 & & \\ \mathrm{H} & 26 & -.9466590 & -2.3355820 & -1.6903710 & 11 & 11 & & & \\ \mathrm{H} & 27 & -3.1541740 & -.9210340 & -1.0982030 & 11 & 12 & & & \\ \mathrm{H} & 28 & -3.0368770 & -.2326140 & 1.5018730 & 11 & 13 & & & \\ \mathrm{H} & 29 & -.7553560 & -1.2364910 & 2.5285250 & 11 & 14 & & & \\ \mathrm{H} & 30 & .7034060 & -.2945750 & -2.4305530 & 11 & 15 & & & \\ \mathrm{H} & 31 & .3008520 & 1.4280950 & -2.1466930 & 11 & 15 & & & \\ \mathrm{H} & 32 & -.9648320 & .2670130 & -2.5627610 & 11 & 15 & & & \\ & & & & & & & & \end{array}$

14

$\begin{array}{lccccccccc}\mathrm{Fe} & 1 & -.5604260 & -.0937740 & .0764070 & 266 & 2 & 4 & 9 & 10 \\ \mathrm{C} & 2 & -.1584710 & .8687150 & 1.4705390 & 62 & 1 & 3 & & \end{array}$ 
(C) 2003 American Chemical Society, Organometallics, Nakazawa om0208319 Supporting Info Page 36

$\begin{array}{lrrrrrrrrr}\mathrm{O} & 3 & .0699100 & 1.4706150 & 2.4372970 & 81 & 2 & & & \\ \mathrm{Si} & 4 & 1.8087000 & .0310290 & -.2038290 & 144 & 1 & 5 & 6 & 7 \\ \mathrm{C} & 5 & 2.3330070 & -.6736770 & -1.8934150 & 64 & 4 & 11 & 12 & 13 \\ \mathrm{C} & 6 & 2.7084010 & -.9666450 & 1.1556140 & 64 & 4 & 14 & 15 & 16 \\ \mathrm{C} & 7 & 2.4950660 & 1.8035120 & -.1083550 & 64 & 4 & 17 & 1.8 & 1.9 \\ \mathrm{C} & 8 & -2.3250010 & 2.5472330 & -.5809400 & 64 & 10 & 20 & 21 & 22 \\ \mathrm{~N} & 9 & -.3222710 & 1.2331000 & -1.5253780 & 72 & 1 & 10 & & \\ \mathrm{C} & 10 & -1.2436130 & 1.5633340 & -.8236900 & 63 & 1 & 8 & 9 & \\ \mathrm{H} & 11 & .3 .4116830 & -.5231800 & -2.0365880 & 11 & 5 & & & \\ \mathrm{H} & 12 & 1.8069270 & -.1555850 & -2.7012350 & 11 & 5 & & & \\ \mathrm{H} & 13 & 2.1356330 & -1.7474770 & -1.9907450 & 11 & 5 & & & \\ \mathrm{H} & 14 & 3.7957580 & -.9084450 & 1.0117120 & 11 & 6 & & & \\ \mathrm{H} & 15 & 2.4341880 & -2.0285340 & 1.1532510 & 11 & 6 & & & \\ \mathrm{H} & 16 & 2.4865610 & -.5690510 & 2.1534420 & 11 & 6 & & & \\ \mathrm{H} & 17 & 3.5809480 & 1.7846490 & -.2713150 & 11 & 7 & & & \\ \mathrm{H} & 18 & 2.3144710 & 2.2672660 & .8679660 & 11 & 7 & & & \\ \mathrm{H} & 19 & 2.0502970 & 2.4419790 & -.8782870 & 11 & 7 & & & \\ \mathrm{H} & 20 & -3.2878570 & 2.0373830 & -.4789300 & 11 & 8 & & & \\ \mathrm{H} & 21 & -2.3790230 & 3.2670870 & -1.4048480 & 11 & 8 & & & \\ \mathrm{H} & 22 & -2.1339350 & 3.0839420 & .3544130 & 11 & 8 & & & \\ \mathrm{C} & 23 & -.3133880 & -2.1935730 & -.2316780 & 63 & 24 & 27 & 28 & \\ \mathrm{C} & 24 & -1.2645960 & -1.7241810 & -1.1758580 & 63 & 23 & 25 & 29 & \\ \mathrm{C} & 25 & -2.3709090 & -1.1790750 & -.4597900 & 63 & 24 & 26 & 30 & \\ \mathrm{C} & 26 & -2.1067600 & -1.3030740 & .9284300 & 63 & 25 & 27 & 31 & \\ \mathrm{C} & 27 & -.8226660 & -1.9208720 & 1.0749090 & 63 & 23 & 26 & 32 & \\ \mathrm{H} & 28 & .6158230 & -2.6948770 & -.4591630 & 11 & 23 & & & \\ \mathrm{H} & 29 & -1.1465530 & -1.7306240 & -2.2508620 & 11 & 24 & & & \\ \mathrm{H} & 30 & -3.2455430 & -.7199120 & -.9017290 & 11 & 25 & & & \\ \mathrm{H} & 31 & -2.7469800 & -.9702200 & 1.7347200 & 11 & 26 & & & \\ \mathrm{H} & 32 & -.3329210 & -2.1574740 & 2.0098920 & 11 & 27 & & & \\ & & & & & & & & & \end{array}$

15

$\begin{array}{lccccccccc}\mathrm{Fe} & 1 & 1.2061500 & .1925120 & -.0183410 & 266 & 2 & 5 & 6 & \\ \mathrm{C} & 2 & 1.2091610 & 1.7532910 & -.8128530 & 62 & 1 & 3 & & \\ \mathrm{O} & 3 & 1.2646520 & 2.7835820 & -1.3460060 & 81 & 2 & & & \\ \mathrm{C} & 4 & -.4543100 & 1.2266370 & 2.5727720 & 64 & 6 & 11 & 12 & 13\end{array}$


(C) 2003 American Chemical Society, Organometallics, Nakazawa om0208319 Supporting Info Page 37

$\begin{array}{lrrrrrrrrr}\mathrm{N} & 5 & -.7923780 & .1320410 & .3273540 & 73 & 1 & 6 & 7 & \\ \mathrm{C} & 6 & -.0920380 & .6583030 & 1.2402460 & 63 & 1 & 4 & 5 & \\ \mathrm{Si} & 7 & -2.3930930 & -.2861410 & -.2779470 & 144 & 5 & 8 & 9 & 10 \\ \mathrm{C} & 8 & -3.7332490 & .1854200 & .9726840 & 64 & 7 & 14 & 15 & 16 \\ \mathrm{C} & 9 & -2.4046140 & -2.1480980 & -.5777990 & 64 & 7 & 17 & 1.8 & 19 \\ \mathrm{C} & 10 & -2.6282170 & .6553790 & -1.8923520 & 64 & 7 & 20 & 21 & 22 \\ \mathrm{H} & 11 & .1392860 & .7343240 & 3.3519130 & 11 & 4 & & & \\ \mathrm{H} & 12 & -1.5202700 & 1.1276340 & 2.8093560 & 11 & 4 & & \\ \mathrm{H} & 13 & -.1747800 & 2.2869220 & 2.5949970 & 11 & 4 & & & \\ \mathrm{H} & 14 & -4.7267870 & -.0730470 & .5843360 & 11 & 8 & & & \\ \mathrm{H} & 15 & -3.7325770 & 1.2619720 & 1.1803480 & 11 & 8 & & & \\ \mathrm{H} & 16 & -3.6058490 & -.3424830 & 1.9252250 & 11 & 8 & & & \\ \mathrm{H} & 17 & -3.3709980 & -2.4746630 & -.9815910 & 11 & 9 & & & \\ \mathrm{H} & 18 & -2.2260330 & -2.7033400 & .3505670 & 11 & 9 & & & \\ \mathrm{H} & 19 & -1.6278520 & -2.4341450 & -1.2958940 & 11 & 9 & & & \\ \mathrm{H} & 20 & -3.5773830 & .3887360 & -2.3735880 & 11 & 10 & & & \\ \mathrm{H} & 21 & -1.8164420 & .4285930 & -2.5926010 & 11 & 10 & & & \\ \mathrm{H} & 22 & -2.6290930 & 1.7387500 & -1.7251400 & 11 & 10 & & & \\ \mathrm{C} & 23 & 2.3292360 & -1.2190980 & -1.2013930 & 63 & & 24 & 27 & 28 \\ \mathrm{C} & 24 & 1.7053940 & -1.9387630 & -.1458350 & 63 & & 23 & 25 & 29 \\ \mathrm{C} & 25 & 2.1605030 & -1.4197680 & 1.0945770 & 63 & & 24 & 26 & 30 \\ \mathrm{C} & 26 & 3.0780800 & -.3588970 & .8221730 & 63 & & 25 & 27 & 31 \\ \mathrm{C} & 27 & 3.1778990 & -.2375470 & -.5956000 & 63 & & 23 & 26 & .32 \\ \mathrm{H} & 28 & 2.1975530 & -1.3866300 & -2.2614500 & 11 & 23 & & & \\ \mathrm{H} & 29 & .9614290 & -2.7158550 & -.2671350 & 11 & 24 & & \\ \mathrm{H} & 30 & 1.8504960 & -1.7570780 & 2.0751300 & 11 & 25 & & \\ \mathrm{H} & 31 & 3.6077620 & .2380400 & 1.5520590 & 11 & 26 & & \\ \mathrm{H} & 32 & 3.7987900 & .4739560 & -1.1249570 & 11 & 27 & & & \\ & & & & & & & & \end{array}$

16

$\begin{array}{lccccccccc}\mathrm{Fe} & 1 & 1.5720390 & .1703380 & .0931260 & 266 & 2 & 5 & 10 & \\ \mathrm{C} & 2 & 1.6145610 & 1.8333660 & -.4380240 & 62 & 1 & 3 & & \\ \mathrm{O} & 3 & 1.6570600 & 2.9413210 & -.7708280 & 81 & 2 & & & \\ \mathrm{~N} & 4 & -1.3990080 & -.0480130 & -.0392150 & 72 & 5 & 6 & & \\ \mathrm{C} & 5 & -.2166120 & .0423640 & -.0014170 & 62 & 1 & 4 & & \\ \mathrm{Si} & 6 & -3.1561300 & -.1299760 & -.0506520 & 144 & 4 & 7 & 8 & 9\end{array}$


(C) 2003 American Chemical Society, Organometallics, Nakazawa om0208319 Supporting Info Page 38

$\begin{array}{lrrrrrrrrr}\mathrm{C} & 7 & -3.7211830 & -.2386650 & 1.7403850 & 64 & 6 & 11 & 12 & 13 \\ \mathrm{C} & 8 & -3.6237740 & -1.6714670 & -1.0239500 & 64 & 6 & 14 & 15 & 16 \\ \mathrm{C} & 9 & -3.7723620 & 1.4392020 & -.8851340 & 64 & 6 & 17 & 18 & 19 \\ \mathrm{C} & 10 & 1.3160650 & .8842400 & 1.9806990 & 64 & 1 & 20 & 21 & 22 \\ \mathrm{H} & 11 & -4.8157630 & -.2787060 & 1.8032980 & 11 & 7 & & & \\ \mathrm{H} & 12 & -3.3833560 & .6313260 & 2.3146170 & 11 & 7 & & & \\ \mathrm{H} & 13 & -3.3240340 & -1.1360960 & 2.2280790 & 11 & 7 & & & \\ \mathrm{H} & 14 & -4.7137910 & -1.7855980 & -1.0714580 & 11 & 8 & & \\ \mathrm{H} & 15 & -3.2136310 & -2.5752780 & -.5591490 & 11 & 8 & & \\ \mathrm{H} & 16 & -3.2477900 & -1.6211860 & -2.0520820 & 11 & 8 & & \\ \mathrm{H} & 17 & -4.8687020 & 1.4567470 & -.9215100 & 11 & 9 & & \\ \mathrm{H} & 18 & -3.4009880 & 1.5147960 & -1.9133240 & 11 & 9 & & \\ \mathrm{H} & 19 & -3.4409400 & 2.3318600 & -.3427310 & 11 & 9 & & & \\ \mathrm{H} & 20 & 1.0113030 & .0493100 & 2.6220010 & 11 & 10 & & & \\ \mathrm{H} & 21 & .5591740 & 1.6722340 & 2.0582010 & 11 & 10 & & & \\ \mathrm{H} & 22 & 2.2699270 & 1.2866460 & 2.3406390 & 11 & 10 & & & \\ \mathrm{C} & 23 & 2.0069340 & -1.5994190 & -1.0835940 & 63 & 24 & 27 & 28 & \\ \mathrm{C} & 24 & 2.0500080 & -1.9252050 & .3141790 & 63 & 23 & 25 & 29 & \\ \mathrm{C} & 25 & 3.0849710 & -1.1542310 & .9045990 & 63 & 24 & 26 & 30 & \\ \mathrm{C} & 26 & 3.6624010 & -.3317100 & -.0976690 & 63 & 25 & 27 & 31 & \\ \mathrm{C} & 27 & 2.9970480 & -.6234650 & -1.3371050 & 63 & 23 & 26 & 32 & \\ \mathrm{H} & 28 & 1.3179220 & -2.0087890 & -1.8105000 & 11 & 23 & & & \\ \mathrm{H} & 29 & 1.4171800 & -2.6403980 & .8219880 & 11 & 24 & & & \\ \mathrm{H} & 30 & 3.3439780 & -1.1443440 & 1.9544640 & 11 & 25 & & & \\ \mathrm{H} & 31 & 4.4704020 & .3737800 & .0418120 & 11 & 26 & & & \\ \mathrm{H} & 32 & 3.2005460 & -.1565830 & -2.2919010 & 11 & 27 & & & \\ & & & & & & & & & \end{array}$

$\begin{array}{lccccccccc}17 & & & & & & & & & \\ \mathrm{Fe} & 1 & .2370910 & .3112580 & -.2515280 & 266 & 2 & 4 & & \\ \mathrm{C} & 2 & 1.8350760 & -.4235960 & -.1083450 & 62 & 1 & 3 & & \\ \mathrm{O} & 3 & 2.8490590 & -.9587190 & .0394320 & 81 & 2 & & & \\ \mathrm{C} & 4 & .9019170 & 2.0969150 & -.0049330 & 64 & 1 & 5 & 6 & 7 \\ \mathrm{H} & 5 & .1785680 & 2.8132650 & .3971870 & 11 & 4 & & & \\ \mathrm{H} & 6 & .9518610 & 2.2375420 & -1.1169740 & 11 & 4 & & & \\ \mathrm{H} . & 7 & 1.8991890 & 2.2979820 & .3939090 & 11 & 4 & & & \\ \mathrm{C} & 8 & -1.5765370 & -.5781590 & -1.0331120 & 63 & 9 & 12 & 13 & \end{array}$


(C) 2003 American Chemical Society, Organometallics, Nakazawa om0208319 Supporting Info Page 39

$\begin{array}{rrrrrrrrr}\mathrm{C} & 9 & -1.8793630 & .5849030 & -.2457880 & 63 & 8 & 10 & 14 \\ \mathrm{C} & 10 & -1.4131820 & .3473390 & 1.0711980 & 63 & 9 & 11 & 15 \\ \mathrm{C} & 11 & -.7871540 & -.9349450 & 1.0938970 & 63 & 10 & 12 & 16 \\ \mathrm{C} & 12 & -.9369930 & -1.5257940 & -.2078730 & 63 & 8 & 11 & 17 \\ \mathrm{H} & 13 & -1.7766270 & -.6947180 & -2.0910570 & 11 & 8 & & \\ \mathrm{H} & 14 & -2.3687660 & 1.4825870 & -.5998570 & 11 & 9 & & \\ \mathrm{H} & 15 & -1.4464940 & 1.0451620 & 1.8966910 & 11 & 10 & & \\ \mathrm{H} & 16 & -.3211740 & -1.3979740 & 1.9532130 & 11 & 11 & & \\ \mathrm{H} & 17 & -.5650670 & -2.4942120 & -.5143990 & 11 & 12 & & \end{array}$

18

$\begin{array}{lrrrrrrrrr}\mathrm{Fe} & 1 & 1.6203930 & .1729290 & .1000950 & 266 & 2 & 5 & 10 \\ \mathrm{C} & 2 & 1.7074000 & 1.8303820 & -.4540480 & 62 & 1 & 3 & & \\ \mathrm{O} & 3 & 1.7956490 & 2.9338740 & -.7945300 & 81 & 2 & & & \\ \mathrm{C} & 4 & -1.4082340 & -.0243440 & -.0162480 & 62 & 5 & 6 & & \\ \mathrm{~N} & 5 & -.2444960 & .0517880 & .0135960 & 72 & 1 & 4 & & \\ \mathrm{Si} & 6 & -3.2798800 & -.1171780 & -.0500170 & 144 & 4 & 7 & 8 & 9 \\ \mathrm{C} & 7 & -3.8557590 & -.2532090 & 1.7379990 & 64 & 6 & 16 & 17 & 18 \\ \mathrm{C} & 8 & -3.7273620 & -1.6476390 & -1.0544100 & 64 & 6 & 19 & 20 & 21 \\ \mathrm{C} & 9 & -3.8816120 & 1.4685600 & -.8691040 & 64 & 6 & 22 & 23 & 24 \\ \mathrm{C} & 10 & 1.3967440 & .9106740 & 1.9719400 & 64 & 1 & 25 & 26 & 27 \\ \mathrm{C} & 11 & 2.0154570 & -1.6483820 & -1.0514620 & 63 & 12 & 15 & 28 & \\ \mathrm{C} & 12 & 2.0846500 & -1.9382110 & .3525680 & 63 & 11 & 13 & 29 & \\ \mathrm{C} & 13 & 3.1294740 & -1.1548750 & .9031200 & 63 & 12 & 14 & 30 & \\ \mathrm{C} & 14 & 3.6847230 & -.3541960 & -.1317810 & 63 & 13 & 15 & 31 & \\ \mathrm{C} & 15 & 3.0034460 & -.6874860 & -1.3532670 & 63 & 11 & 14 & 32 & \\ \mathrm{H} & 16 & -4.9509340 & -.2982580 & 1.7847170 & 11 & 7 & & & \\ \mathrm{H} & 17 & -3.5293950 & .6102150 & 2.3280230 & 11 & 7 & & & \\ \mathrm{H} & 18 & -3.4616620 & -1.1560000 & 2.2175180 & 11 & 7 & & & \\ \mathrm{H} & 19 & -4.8170690 & -1.7612020 & -1.1091770 & 11 & 8 & & & \\ \mathrm{H} & 20 & -3.3191330 & -2.5581970 & -.6019640 & 11 & 8 & & & \\ \mathrm{H} & 21 & -3.3461830 & -1.5790440 & -2.0792400 & 11 & 8 & & & \\ \mathrm{H} & 22 & -4.9777450 & 1.4843370 & -.9107910 & 11 & 9 & & & \\ \mathrm{H} & 23 & -3.5048730 & 1.5574130 & -1.8939530 & 11 & 9 & & & \\ \mathrm{H} & 24 & -3.5545120 & 2.3536270 & -.3124050 & 11 & 9 & & & \\ \mathrm{H} & 25 & 1.0777810 & .0791540 & 2.6127690 & 11 & 10 & & & \\ & . & & & & & & & & \end{array}$


(C) 2003 American Chemical Society, Organometallics, Nakazawa om0208319 Supporting Info Page 40

$\begin{array}{rrrrrrc}\mathrm{H} & 26 & .6329360 & 1.6953560 & 2.0216330 & 11 & 10 \\ \mathrm{H} & 27 & 2.3396450 & 1.3188010 & 2.3542740 & 11 & 10 \\ \mathrm{H} & 28 & 1.3023700 & -2.0657970 & -1.7506160 & 11 & 11 \\ \mathrm{H} & 29 & 1.4513470 & -2.6291530 & .8928040 & 11 & 12 \\ \mathrm{H} & 30 & 3.4098230 & -1.1175570 & 1.9468890 & 11 & 13 \\ \mathrm{H} & 31 & 4.5015230 & .3473800 & -.0279310 & 11 & 14 \\ \mathrm{H} & 32 & 3.1908260 & -.2486090 & -2.3245970 & 11 & 15\end{array}$

$\begin{array}{lrrrrrrrrrr}19 & & & & & & & & & & \\ \mathrm{Fe} & 1 & -1.6377850 & .3611970 & -.1843880 & 266 & 2 & 4 & & \\ \mathrm{C} & 2 & -1.5556950 & 2.2958170 & .0426580 & 64 & 1 & 9 & 10 & 11 \\ \mathrm{Si} & 3 & 3.2335240 & -.0964160 & .0062710 & 144 & 5 & 6 & 7 & 8 \\ \mathrm{~N} & 4 & .2084380 & .2016270 & -.0897530 & 72 & 1 & 5 & & \\ \mathrm{C} & 5 & 1.3714490 & .0767050 & -.0593300 & 62 & 3 & 4 & & \\ \mathrm{C} & 6 & 3.6428600 & -1.8923480 & -.3937620 & 64 & 3 & 12 & 13 & 14 \\ \mathrm{C} & 7 & 3.7683470 & .3706120 & 1.7517300 & 64 & 3 & 15 & 16 & 17 \\ \mathrm{C} & 8 & 3.9269540 & 1.0855740 & -1.2868930 & 64 & 3 & 18 & 19 & 20 \\ \mathrm{H} & 9 & -1.3871330 & 2.5845670 & -1.0188270 & 11 & 2 & & & \\ \mathrm{H} & 10 & -.7099350 & 2.6901100 & .6186470 & 11 & 2 & & & \\ \mathrm{H} & 11 & -2.4858160 & 2.7706030 & .3762830 & 11 & 2 & & & \\ \mathrm{H} & 12 & 4.7277480 & -2.0526550 & -.3637430 & 11 & 6 & & & \\ \mathrm{H} & 13 & 3.2906370 & -2.1685070 & -1.3937640 & 11 & 6 & . & & \\ \mathrm{H} & 14 & 3.1835200 & -2.5781910 & .3267320 & 11 & 6 & & & \\ \mathrm{H} & 15 & 4.8583740 & .2950670 & 1.8507890 & 11 & 7 & & & \\ \mathrm{H} & 16 & 3.3171370 & -.2917790 & 2.4987220 & 11 & 7 & & & \\ \mathrm{H} & 17 & 3.4785880 & 1.3986650 & 1.9949200 & 11 & 7 & & & \\ \mathrm{H} & 18 & 5.0228260 & 1.0369280 & -1.2987260 & 11 & 8 & & & \\ \mathrm{H} & 19 & 3.6386910 & 2.1212850 & -1.0766110 & 11 & 8 & & & \\ \mathrm{H} & 20 & 3.5693930 & .8336450 & -2.2913960 & 11 & 8 & & & \\ \mathrm{C} & 21 & -2.1843170 & -1.7531310 & -.0662250 & 63 & 22 & 25 & 26 & \\ \mathrm{C} & 22 & -2.9871060 & -1.1012210 & -1.0263830 & 63 & 21 & 23 & 27 & \\ \mathrm{C} & 23 & -3.6981530 & -.0319830 & -.3820890 & 63 & 22 & 24 & 28 & \\ \mathrm{C} & 24 & -3.3127050 & -.0264690 & .9853420 & 63 & 23 & 25 & 29 & \\ \mathrm{C} & 25 & -2.3468790 & -1.0607780 & 1.1787240 & 63 & 21 & 24 & 30 & \\ \mathrm{H} & 26 & -1.4990420 & -2.5688550 & -.2562690 & 11 & 21 & & & \\ \mathrm{H} & 27 & -3.0330680 & -1.3425960 & -2.0814860 & 11 & 22 & . & & \\ & & & & & & & & \\ & & \end{array}$

